\title{
Position tracking in delayed bilateral teleoperators without velocity measurements
}

\author{
Ioannis Sarras ${ }^{1}$, Emmanuel Nuño $^{2 *}$, Luis Basañez ${ }^{3}$ and Michel Kinnaert ${ }^{4}$ \\ ${ }^{1}$ Automatic Control Department, SUPELEC. Gif-sur-Yvette, France. \\ 2 Department of Computer Science, University of Guadalajara. Guadalajara, Mexico. \\ ${ }^{3}$ Institute of Industrial and Control Engineering, Technical University of Catalonia. Barcelona, Spain. \\ ${ }^{4}$ Control Engineering and Systems Analysis Department, Université Libre de Bruxelles. Brussels, Belgium.
}

\begin{abstract}
SUMMARY
This work considers the control of nonlinear bilateral teleoperators with variable time-delays without the need of velocity measurements. The recently proposed Immersion and Invariance (I\&I) observer is used to obtain an exponentially convergent estimate of the unmeasured velocities. Under the classical assumption that the human operator and the environment define passive, velocity to force, maps it is proved that with this observer together with a Proportional plus damping $(\mathrm{P}+\mathrm{d})$ controller, velocities and position error are globally bounded. Finally, in the case that the human operator and the environment do not exert forces on the local and remote manipulators, respectively, global asymptotic convergence of velocities and of position error to zero is achieved. The theoretical results are sustained with simulations using a couple of two degreesof-freedom nonlinear manipulators.
\end{abstract}

KEY WORDS: Nonlinear Control Systems, Output Regulation, Bilateral Teleoperators, Time-Delays,

Velocity Observers.

\section{INTRODUCTION}

In bilateral teleoperators, the local and remote manipulators are interconnected through a communication channel that often imposes time-delays in the transmitted signals. Controlling these systems has become a highly active research field. It is recognized that Anderson and Spong [1] provide the first delay independent controller by mimicking a lossless transmission line for constant time-delays via the scattering transformation. Later, Niemeyer and Slotine [2] introduce the wave variables, following the former scattering approach, and propose a method to eliminate wave reflections. These developments paved the way for the recent proposals on passivity-based controllers. For a recent historical survey on this research line, the reader may refer to [3] and for a stability tutorial to [4]. However, the classic scattering transformation, first conceived to deal

*Correspondence to: 1500 Revolución Av., 'M' Building. 44830, Guadalajara, México. Tel.: +523313785900, Ext.:27748. E-mail: emmanuel.nuno@cucei.udg.mx 
only with constant time-delays, may give rise to position drift. Using the scattering transformation, Chopra et al. [5] propose to send the local position to the remote controller and to add a proportional position error term in the remote controller to ensure position tracking for constant time-delays. Lee and Spong report in [6] a Proportional Derivative plus damping controller that, without employing the scattering transformation, is able to ensure position tracking and asymptotic convergence of velocities to zero, with the stability proof presented in [7]. In [8, ?] an adaptive controller that also ensures position tracking is presented, and in $[9,10]$ its use is extended to the more general case of synchronization of networks of Euler-Lagrange (EL) systems. It is in [11] where the proof that simple Proportional plus damping $(\mathrm{P}+\mathrm{d})$ controllers can ensure position tracking and asymptotic convergence of velocities to zero in the presence of variable time-delays without the incorporation of the scattering transformation is given. The $\mathrm{P}+\mathrm{d}$ controllers have also been used in the consensus control of networked EL-systems in [12] and their robustness to parameter uncertainty has been analyzed in [13].

The aforementioned schemes and most of the previous works (e.g., $[14,15,16,17])$ have tackled the problem of controlling a teleoperation system assuming that velocities are measurable. However, most of the commercially available robots, that can be used in teleoperation systems, are not equipped with velocity sensors, mainly to save cost, space and weight. Few remarkable exceptions [18, 19] and more recently [20] and [21] have dealt with this problem. In [18] a sliding control technique is used to control a linearized version of the local and remote manipulators, making use of measurements of the force of the human and of the environment. An adaptive control scheme in order to render the teleoperator Input-to-State stable is provided in [19]. The work of [20] proves boundedness of the position error using a high-gain velocity observer. Finally, the work reported in [21] proposes the use of a dynamic controller that back-propagates damping and can achieve position tracking for teleoperators without delays.

Recently, a full-order globally exponentially convergent velocity observer has been proposed for a general class of mechanical systems with or without constraints [22, 23]. The adopted approach is based on the notions of Immersion and Invariance (I\&I), where the objective consists in finding a certain manifold $\mathcal{M}$, in the extended state-space of the plant and the observer, that should be rendered attractive and invariant. Moreover, since a nonlinear partial differential equation (PDE) needs to be solved, the authors propose an "approximation" technique in order to avoid the need of solving this PDE. Finally, in order to provide a Lyapunov-based stability analysis, a dynamic scaling as well as some high-gain terms are introduced, see also [24, 25]. Another approach based on I\&I has been also recently proposed in [26], and presents a constructive solution to the aforementioned PDE for the case of mechanical systems that can be rendered linear in the velocities via a (partial) change of coordinates. It shows that the defined linearizable class strictly contains the class studied in the existing literature on linearization for speed observation or control, and a reduced order globally exponentially stable observer is reported. Moreover, the combination of the proposed reduced order I\&I observer with an Interconnection and Damping Assignment Passivity-Based Control (IDA-PBC) law was proved to ensure the asymptotic stability of the desired equilibrium.

The main contributions of this paper are: 1) the extension of the authors' previous $\mathrm{P}+\mathrm{d}$ controllers for variable time-delays [11] to the more challenging case when velocity measurements are not available; 2) it is proved, under the commonly adopted assumption that the human operator and the environment define passive maps, from velocity to force, that when the bilateral teleoperator 
is in closed-loop with the P+d controller and the full-order I\&I observer [22] is used to find a velocity estimation, position error and velocities are globally bounded. Moreover, it is also shown that, if the human operator and the environment do not exert any forces on the local and remote manipulators then position error and velocities asymptotically converge to zero; 3 ) the design, different from previous works, in particular to [27], of a velocity observer for general nonlinear manipulators interconnected with a communication channel that can induce variable time-delays. Up to the authors knowledge, this is among the first works that can guarantee position tracking capabilities in bilateral teleoperators that are not equipped with velocity sensors and that exhibit variable time-delays in the communication channel.

\section{BILATERAL TELEOPERATION SYSTEM}

To streamline the presentation, throughout the paper the following notation is introduced. Lower case letters denote scalar functions, e.g. $t$, bold lower case letters denote vectors, e.g. $\mathbf{x}$, and bold upper case letters denote matrices, e.g. A. Moreover, I, $\varnothing$ will be the identity and all-zero matrices, respectively, of appropriate dimensions. Additionally, we define $\mathbb{R}:=(-\infty, \infty), \mathbb{R}_{>0}:=$ $(0, \infty), \mathbb{R}_{\geq 0}:=[0, \infty) . \lambda_{m}\{\mathbf{A}\}$ and $\lambda_{M}\{\mathbf{A}\}$ represent the minimum and maximum eigenvalues of matrix $\mathbf{A}$, respectively while $\|\mathbf{A}\|$ denotes the matrix-induced 2-norm. $|\mathbf{x}|$ stands for the standard Euclidean norm of vector $\mathbf{x}$. For any function $\mathbf{f}: \mathbb{R}_{\geq 0} \rightarrow \mathbb{R}^{n}$, the $\mathcal{L}_{\infty}$-norm is defined as $\|\mathbf{f}\|_{\infty}:=\sup _{t \geq 0}|\mathbf{f}(t)|$, and the $\mathcal{L}_{2}$-norm as $\|\mathbf{f}\|_{2}:=\left(\int_{0}^{\infty}|\mathbf{f}(t)|^{2} d t\right)^{\frac{1}{2}}$. The $\mathcal{L}_{\infty}$ and $\mathcal{L}_{2}$ spaces are defined as the sets $\left\{\mathbf{f}: \mathbb{R}_{\geq 0} \rightarrow \mathbb{R}^{n}:\|\mathbf{f}\|_{\infty}<\infty\right\}$ and $\left\{\mathbf{f}: \mathbb{R}_{\geq 0} \rightarrow \mathbb{R}^{n}:\|\mathbf{f}\|_{2}<\infty\right\}$, respectively.

The following lemma, borrowed from [11], serves as instrumental in the proof of the main result of the paper.

\section{Lemma 1}

[11]. For any vector signals $\mathbf{x}, \mathbf{y} \in \mathbb{R}^{n}$, any variable time-delay $0 \leq T(t) \leq{ }^{*} T<\infty$ and any constant $\alpha>0$, the following inequality holds

$$
-\int_{0}^{t} \mathbf{x}^{\top}(\sigma) \int_{-T(\sigma)}^{0} \mathbf{y}(\sigma+\theta) d \theta d \sigma \leq \frac{\alpha}{2}\|\mathbf{x}\|_{2}^{2}+\frac{{ }^{*} T^{2}}{2 \alpha}\|\mathbf{y}\|_{2}^{2},
$$

where $\|\cdot\|_{2}$ stands for the $\mathcal{L}_{2}$-norm.

\subsection{Nonlinear Dynamical Model}

The local and remote manipulators are modeled as a pair of $n$-Degree Of Freedom (DOF) serial links with revolute joints. Their corresponding nonlinear dynamics are described by

$$
\begin{aligned}
\mathbf{M}_{l}\left(\mathbf{q}_{l}\right) \ddot{\mathbf{q}}_{l}+\mathbf{C}_{l}\left(\mathbf{q}_{l}, \dot{\mathbf{q}}_{l}\right) \dot{\mathbf{q}}_{l}+\mathbf{g}_{l}\left(\mathbf{q}_{l}\right) & =\boldsymbol{\tau}_{h}-\boldsymbol{\tau}_{l} \\
\mathbf{M}_{r}\left(\mathbf{q}_{r}\right) \ddot{\mathbf{q}}_{r}+\mathbf{C}_{r}\left(\mathbf{q}_{r}, \dot{\mathbf{q}}_{r}\right) \dot{\mathbf{q}}_{r}+\mathbf{g}_{r}\left(\mathbf{q}_{r}\right) & =\boldsymbol{\tau}_{r}-\boldsymbol{\tau}_{e}
\end{aligned}
$$

where $\ddot{\mathbf{q}}_{i}, \dot{\mathbf{q}}_{i}, \mathbf{q}_{i} \in \mathbb{R}^{n}$ are the acceleration, the velocity and the joint position, respectively. The mappings $\mathbf{M}_{i}: \mathbb{R}^{n} \rightarrow \mathbb{R}^{n \times n}$ define the inertia matrices, $\mathbf{C}_{i}: \mathbb{R}^{n} \times \mathbb{R}^{n} \rightarrow \mathbb{R}^{n \times n}$ the Coriolis and centrifugal effects, defined via the Christoffel symbols of the first kind, $\mathbf{g}_{i}: \mathbb{R}^{n} \rightarrow \mathbb{R}^{n}$ the vectors of 
gravitational forces, $\boldsymbol{\tau}_{i} \in \mathbb{R}^{n}$ are the control signals and $\boldsymbol{\tau}_{h} \in \mathbb{R}^{n}, \boldsymbol{\tau}_{e} \in \mathbb{R}^{n}$ are the joint torques corresponding to the forces exerted by the human operator and the environment interaction, respectively. The subscript $i=\{l, r\}$ refers to the local and remote manipulator, respectively.

In order to express dynamics (1) in a more compact way, we define the concatenated positions of the remote and local manipulators as $\mathbf{q}=\operatorname{col}\left(\mathbf{q}_{l}^{\top}, \mathbf{q}_{r}^{\top}\right)$, the total inertia and Coriolis matrices as $\mathbf{M}(\mathbf{q})=\operatorname{diag}\left(\mathbf{M}_{l}\left(\mathbf{q}_{l}\right), \mathbf{M}_{r}\left(\mathbf{q}_{r}\right)\right)$ and $\mathbf{C}(\mathbf{q}, \dot{\mathbf{q}})=\operatorname{diag}\left(\mathbf{C}_{l}\left(\mathbf{q}_{l}, \dot{\mathbf{q}}_{l}\right), \mathbf{C}_{r}\left(\mathbf{q}_{r}, \dot{\mathbf{q}}_{r}\right)\right)$, while the (local and remote) control and external (human and environmental) forces are denoted, respectively, by $\boldsymbol{\tau}=\operatorname{col}\left(-\boldsymbol{\tau}_{l}^{\top}, \boldsymbol{\tau}_{r}^{\top}\right)$ and $\mathbf{w}=\operatorname{col}\left(\boldsymbol{\tau}_{h}^{\top},-\boldsymbol{\tau}_{e}^{\top}\right)$. Hence, the teleoperator dynamics is compactly written as

$$
\mathbf{M}(\mathbf{q}) \ddot{\mathbf{q}}+\mathbf{C}(\mathbf{q}, \dot{\mathbf{q}}) \dot{\mathbf{q}}+\mathbf{g}(\mathbf{q})=\tau+\mathbf{w} .
$$

In this work, the following standard assumptions are adopted:

A1. The generalized inertia matrix is positive definite and bounded, that is, $m_{m} \mathbf{I} \leq \mathbf{M}(\mathbf{q}) \leq m_{M} \mathbf{I}$ $\forall \mathbf{q}$, where $m_{m}:=\lambda_{\mathrm{m}}\{\mathbf{M}(\mathbf{q})\}$ and $m_{M}:=\lambda_{\mathrm{M}}\{\mathbf{M}(\mathbf{q})\}$.

A2. The human operator and the environment define passive, velocity to force, maps. Hence, $\exists \kappa_{i} \in \mathbb{R}_{\geq 0}$, such that, $\forall t \geq 0$,

$$
E_{h}:=-\int_{0}^{t} \dot{\mathbf{q}}_{l}^{\top} \mathbf{K}_{h} \boldsymbol{\tau}_{h} d \sigma+\kappa_{l} \geq 0 \quad E_{e}:=\int_{0}^{t} \dot{\mathbf{q}}_{r}^{\top} \mathbf{K}_{e} \boldsymbol{\tau}_{e} d \sigma+\kappa_{r} \geq 0
$$

where $\mathbf{K}_{h}$ and $\mathbf{K}_{e}$ are diagonal and positive definite scale matrices.

A3. The variable time-delay has a known upper bound ${ }^{*} T_{i}$. i.e., $0 \leq T_{i}(t) \leq{ }^{*} T_{i}<\infty$.

It is well known that dynamics (1) enjoy the following properties [28, 29, 11]:

P1. For all $\mathbf{a} \in \mathbb{R}^{2 n}, \mathbf{a}^{\top}[\dot{\mathbf{M}}(\mathbf{q})-2 \mathbf{C}(\mathbf{q}, \dot{\mathbf{q}})] \mathbf{a}=0$.

P2. For all $\mathbf{q}, \mathbf{a}, \mathbf{b}, \mathbf{c} \in \mathbb{R}^{2 n}$ and any scalars $\alpha_{1}, \alpha_{2}$ we have

$$
\begin{gathered}
\mathbf{C}(\mathbf{q}, \mathbf{a}) \mathbf{b}=\mathbf{C}(\mathbf{q}, \mathbf{b}) \mathbf{a} \\
\mathbf{C}\left(\mathbf{q}, \alpha_{1} \mathbf{c}+\alpha_{2} \mathbf{a}\right) \mathbf{b}=\alpha_{1} \mathbf{C}(\mathbf{q}, \mathbf{c}) \mathbf{b}+\alpha_{2} \mathbf{C}(\mathbf{q}, \mathbf{a}) \mathbf{b}
\end{gathered}
$$

P3. For all $\mathbf{q}, \mathbf{a}, \mathbf{b} \in \mathbb{R}^{2 n}, \exists k_{c} \in \mathbb{R}_{>0}$ such that $|\mathbf{C}(\mathbf{q}, \mathbf{a}) \mathbf{b}| \leq k_{c}|\mathbf{a}||\mathbf{b}|$.

P4. If Assumption $\mathrm{A} 1$ holds and $\dot{\mathbf{q}}, \ddot{\mathbf{q}} \in \mathcal{L}_{\infty}$ then $\frac{d}{d t} \mathbf{C}(\mathbf{q}, \dot{\mathbf{q}})$ is a bounded operator.

Let be the following factorization of the inertia matrix, borrowed from [22]:

$$
\mathbf{M}(\mathbf{q})=\Psi^{\top}(\mathbf{q}) \Psi(\mathbf{q})
$$

where the mapping $\Psi: \mathbb{R}^{2 n} \rightarrow \mathbb{R}^{2 n \times 2 n}$ is given by

$$
\mathbf{\Psi}(\mathbf{q}):=\operatorname{diag}\left(\mathbf{\Psi}_{l}\left(\mathbf{q}_{l}\right), \mathbf{\Psi}_{r}\left(\mathbf{q}_{r}\right)\right),
$$

and $\boldsymbol{\Psi}_{i}: \mathbb{R}^{n} \rightarrow \mathbb{R}^{n \times n}$ are the mappings corresponding to factorizations of the inertia matrices $\mathbf{M}_{i}=\boldsymbol{\Psi}_{i}^{\top}\left(\mathbf{q}_{i}\right) \boldsymbol{\Psi}_{i}\left(\mathbf{q}_{i}\right)$. Since $\mathbf{M}_{i}\left(\mathbf{q}_{i}\right)$ is symmetric and it satisfies A1, $\boldsymbol{\Psi}_{i}\left(\mathbf{q}_{i}\right)$ always exists. Further, let the mappings $\mathbf{L}: \mathbb{R}^{2 n} \rightarrow \mathbb{R}^{2 n \times 2 n}$ and $\mathbf{F}: \mathbb{R}^{2 n} \times \mathbb{R}^{2 n} \times \mathbb{R}^{2 n} \rightarrow \mathbb{R}^{2 n}$ be defined as

$$
\mathbf{L}(\mathbf{q})=\Psi^{-1}(\mathbf{q}), \quad \mathbf{F}(\mathbf{q}, \boldsymbol{\tau}, \mathbf{w})=\mathbf{L}^{\top}(\mathbf{q})(\boldsymbol{\tau}+\mathbf{w}-\mathbf{g}(\mathbf{q})),
$$


and consider the following coordinate transformation

$$
\mathrm{x}:=\Psi(\mathbf{q}) \dot{\mathbf{q}}
$$

Then, using (3) and (4), dynamics (1) can be transformed into the new system

$$
\begin{aligned}
\dot{\mathbf{q}} & =\mathbf{L}(\mathbf{q}) \mathbf{x} \\
\dot{\mathbf{x}} & =\mathbf{S}(\mathbf{q}, \mathbf{x}) \mathbf{x}+\mathbf{F}(\mathbf{q}, \boldsymbol{\tau}, \mathbf{w}),
\end{aligned}
$$

with the mapping $\mathbf{S}: \mathbb{R}^{2 n} \times \mathbb{R}^{2 n} \rightarrow \mathbb{R}^{2 n \times 2 n}$ defined as

$$
\mathbf{S}(\mathbf{q}, \mathbf{x}):=\left[\dot{\Psi}(\mathbf{q})-\Psi^{-\top}(\mathbf{q}) \mathbf{C}(\mathbf{q}, \mathbf{x})\right] \Psi^{-1}(\mathbf{q})
$$

The mapping $\mathbf{S}$ has the following properties, related to the properties of the Coriolis matrix $\mathbf{C}$ $[22,23]$, that are useful for the derivations:

P5. $\mathbf{S}(\mathbf{q}, \mathbf{x})$ is skew-symmetric, i.e., $\mathbf{S}=-\mathbf{S}^{\top}$.

P6. $\mathbf{S}(\mathbf{q}, \mathbf{x})$ is linear in the second argument, i.e., $\mathbf{S}\left(\mathbf{q}, \alpha_{1} \mathbf{c}+\alpha_{2} \mathbf{a}\right) \mathbf{b}=\alpha_{1} \mathbf{S}(\mathbf{q}, \mathbf{c}) \mathbf{b}+\alpha_{2} \mathbf{S}(\mathbf{q}, \mathbf{a}) \mathbf{b}$, for all $\mathbf{q}, \mathbf{a}, \mathbf{b}, \mathbf{c} \in \mathbb{R}^{2 n}$ and any scalars $\alpha_{1}, \alpha_{2}$.

P7. There exists a mapping $\overline{\mathbf{S}}: \mathbb{R}^{2 n} \times \mathbb{R}^{2 n} \rightarrow \mathbb{R}^{2 n \times 2 n}$ satisfying $\mathbf{S}(\mathbf{q}, \mathbf{x}) \mathbf{z}=\overline{\mathbf{S}}(\mathbf{q}, \mathbf{z}) \mathbf{x}$, for all $\mathbf{q}, \mathbf{x}, \mathbf{z} \in \mathbb{R}^{2 n}$.

\section{IMMERSION AND INVARIANCE OBSERVER}

Let us briefly remind some basic points concerning the observer design. For further details the interested reader should consult $[22,24]$. For system (5), it is proposed the manifold

$$
\mathcal{M}=\{(\mathbf{q}, \mathbf{x}, \boldsymbol{\xi}, \hat{\mathbf{x}}, \hat{\mathbf{q}}: \boldsymbol{\xi}+\boldsymbol{\beta}(\mathbf{q}, \hat{\mathbf{q}}, \hat{\mathbf{x}})-\mathbf{x}=\mathbf{0}\}
$$

where $\boldsymbol{\xi} \in \mathbb{R}^{2 n}, \hat{\mathbf{q}} \in \mathbb{R}^{2 n}$ and $\hat{\mathbf{x}} \in \mathbb{R}^{2 n}$ are (part of) the observer state, whose dynamics, as well as the mapping $\boldsymbol{\beta} \in \mathbb{R}^{2 n} \times \mathbb{R}^{2 n} \times \mathbb{R}^{2 n} \rightarrow \mathbb{R}^{2 n}$, are defined below. To prove that the manifold $\mathcal{M}$ is attractive and invariant, it is shown that the off-the-manifold coordinate

$$
\mathbf{z}=\boldsymbol{\xi}+\boldsymbol{\beta}(\mathbf{q}, \hat{\mathbf{q}}, \hat{\mathbf{x}})-\mathbf{x}
$$

whose norm determines the distance of the state from the manifold $\mathcal{M}$, is such that:

$\mathbf{C} 1$ (Invariance) $\mathbf{z}(0)=0 \Rightarrow \mathbf{z}(t)=0$, for all $t \geq 0$

$\mathrm{C} 2$ (Attractivity) $\mathbf{z}(t)$ asymptotically (exponentially) converges to zero.

Then, if $|\mathbf{z}(t)| \rightarrow 0$, an asymptotic estimate of $\mathbf{x}$ is given by $\boldsymbol{\xi}+\boldsymbol{\beta}$. Convergence to the manifold $\mathcal{M}$ can be proved by examining the $\mathbf{z}$-dynamical behavior that is given by

$$
\begin{aligned}
\dot{\mathbf{z}} & =\dot{\boldsymbol{\xi}}+\dot{\boldsymbol{\beta}}-\dot{\mathbf{x}} \\
& =\dot{\boldsymbol{\xi}}+\nabla_{\mathbf{q}} \boldsymbol{\beta} \mathbf{L}(\mathbf{q}) \mathbf{x}+\nabla_{\hat{\mathbf{q}}} \boldsymbol{\beta} \dot{\hat{\mathbf{q}}}+\nabla_{\hat{\mathbf{x}}} \boldsymbol{\beta} \dot{\hat{\mathbf{x}}}-\mathbf{S}(\mathbf{q}, \mathbf{x}) \mathbf{x}-\mathbf{F}(\mathbf{q}, \boldsymbol{\tau}, \mathbf{w}) .
\end{aligned}
$$


Defining

$$
\dot{\boldsymbol{\xi}}:=\mathbf{F}(\mathbf{q}, \boldsymbol{\tau}, \mathbf{w})-\nabla_{\hat{\mathbf{q}}} \boldsymbol{\beta} \dot{\hat{\mathbf{q}}}-\nabla_{\hat{\mathbf{x}}} \boldsymbol{\beta} \dot{\hat{\mathbf{x}}}+\mathbf{S}(\mathbf{q}, \boldsymbol{\xi}+\boldsymbol{\beta})(\boldsymbol{\xi}+\boldsymbol{\beta})-\nabla_{\mathbf{q}} \boldsymbol{\beta} \mathbf{L}(\mathbf{q})(\boldsymbol{\xi}+\boldsymbol{\beta}),
$$

together with Properties P6 and P7, yields

$$
\dot{\mathbf{z}}=\left[\mathbf{S}(\mathbf{q}, \mathbf{x})+\overline{\mathbf{S}}(\mathbf{q}, \boldsymbol{\xi}+\boldsymbol{\beta})-\nabla_{\mathbf{q}} \boldsymbol{\beta} \mathbf{L}(\mathbf{q})\right] \mathbf{z}
$$

The desired objective consists in finding a certain mapping $\boldsymbol{\beta}$ such that the $\mathbf{z}$-dynamics reduces to $\dot{\mathbf{z}}=[\mathbf{S}(\mathbf{q}, \mathbf{x})-k \mathbf{I}] \mathbf{z}$, where $k \in \mathbb{R}_{>0}$, which ensures $\mathbf{z}=\mathbf{0}$ to be Globally Exponentially Stable (GES). That is, a mapping $\boldsymbol{\beta}$ that solves the partial differential equation (PDE)

$$
\nabla_{\mathbf{q}} \boldsymbol{\beta}=[k \mathbf{I}+\overline{\mathbf{S}}(\mathbf{q}, \boldsymbol{\xi}+\boldsymbol{\beta})] \mathbf{L}^{-1}(\mathbf{q})
$$

However, in general, such a $\boldsymbol{\beta}$ may not exist. Hence, an approximate solution has been proposed by defining an ideal $\nabla_{\mathbf{q}} \boldsymbol{\beta}$ as

$$
\mathbf{H}(\mathbf{q}, \boldsymbol{\xi}+\boldsymbol{\beta}):=[k \mathbf{I}+\overline{\mathbf{S}}(\mathbf{q}, \boldsymbol{\xi}+\boldsymbol{\beta})] \mathbf{L}^{-1}(\mathbf{q}),
$$

and

$$
\boldsymbol{\beta}(\mathbf{q}, \hat{\mathbf{q}}, \hat{\mathbf{x}}):=\mathbf{H}(\hat{\mathbf{q}}, \hat{\mathbf{x}}) \mathbf{q}
$$

The above choices yield

$$
\begin{aligned}
\nabla_{\mathbf{q}} \boldsymbol{\beta}(\mathbf{q}, \hat{\mathbf{q}}, \hat{\mathbf{x}}) & =\mathbf{H}(\mathbf{q}, \boldsymbol{\xi}+\boldsymbol{\beta})-[\mathbf{H}(\mathbf{q}, \boldsymbol{\xi}+\boldsymbol{\beta})-\mathbf{H}(\hat{\mathbf{q}}, \hat{\mathbf{x}})] \\
& =\mathbf{H}(\mathbf{q}, \boldsymbol{\xi}+\boldsymbol{\beta})-\boldsymbol{\Delta}_{\mathbf{q}}\left(\mathbf{q}, \hat{\mathbf{x}}, \mathbf{e}_{\mathbf{q}}\right)-\boldsymbol{\Delta}_{\mathbf{x}}\left(\mathbf{q}, \hat{\mathbf{x}}, \mathbf{e}_{\mathbf{x}}\right)
\end{aligned}
$$

where for the second line the definitions

$$
\mathbf{e}_{\mathbf{q}}:=\hat{\mathbf{q}}-\mathbf{q}, \quad \mathbf{e}_{\mathbf{x}}:=\hat{\mathbf{x}}-(\boldsymbol{\xi}+\boldsymbol{\beta})
$$

are used, as well as the fact that mappings $\boldsymbol{\Delta}_{\mathbf{q}}, \boldsymbol{\Delta}_{\mathbf{x}}: \mathbb{R}^{2 n} \times \mathbb{R}^{2 n} \times \mathbb{R}^{2 n} \rightarrow \mathbb{R}^{2 n \times 2 n}$ always exist and are such that for all $\mathbf{q}, \mathbf{x}, \hat{\mathbf{q}}, \hat{\mathbf{x}} \in \mathbb{R}^{2 n}$

$$
\Delta_{\mathbf{q}}(\mathbf{q}, \hat{\mathbf{x}}, \mathbf{0})=\mathbf{0}, \quad \boldsymbol{\Delta}_{\mathbf{x}}(\mathbf{q}, \hat{\mathbf{x}}, \mathbf{0})=\mathbf{0}
$$

Substituting (10) and (12) in (9), yields

$$
\dot{\mathbf{z}}=[\mathbf{S}(\mathbf{q}, \mathbf{x})-k \mathbf{I}] \mathbf{z}+\left[\boldsymbol{\Delta}_{\mathbf{q}}\left(\mathbf{q}, \hat{\mathbf{x}}, \mathbf{e}_{\mathbf{q}}\right)+\boldsymbol{\Delta}_{\mathbf{x}}\left(\mathbf{q}, \hat{\mathbf{x}}, \mathbf{e}_{\mathbf{x}}\right)\right] \mathbf{L}(\mathbf{q}) \mathbf{z}
$$

The mappings $\Delta_{\mathrm{q}}, \Delta_{\mathrm{x}}$ play the role of disturbances that are dominated with a dynamic scaling, with factor $\frac{1}{r}$,

$$
\boldsymbol{\eta}=\frac{1}{r} \mathbf{z}
$$


and a proper choice of the observer dynamics. From the dynamic scaling, the dynamical behavior of $\boldsymbol{\eta}$ is given by

$$
\dot{\boldsymbol{\eta}}=[\mathbf{S}(\mathbf{q}, \mathbf{x})-k \mathbf{I}] \boldsymbol{\eta}+\left[\boldsymbol{\Delta}_{\mathbf{q}}\left(\mathbf{q}, \hat{\mathbf{x}}, \mathbf{e}_{\mathbf{q}}\right)+\boldsymbol{\Delta}_{\mathbf{x}}\left(\mathbf{q}, \hat{\mathbf{x}}, \mathbf{e}_{\mathbf{x}}\right)\right] \mathbf{L}(\mathbf{q}) \boldsymbol{\eta}-\frac{\dot{r}}{r} \boldsymbol{\eta}
$$

Using $V_{1}=\frac{1}{2}|\boldsymbol{\eta}|^{2}$, setting the following $r$-dynamics

$$
\dot{r}=-\frac{k}{4}(r-1)+\frac{r}{k}\left(\left\|\boldsymbol{\Delta}_{\mathbf{q}}\left(\mathbf{q}, \hat{\mathbf{x}}, \mathbf{e}_{\mathbf{q}}\right) \mathbf{L}(\mathbf{q})\right\|^{2}+\left\|\boldsymbol{\Delta}_{\mathbf{x}}\left(\mathbf{q}, \hat{\mathbf{x}}, \mathbf{e}_{\mathbf{x}}\right) \mathbf{L}(\mathbf{q})\right\|^{2}\right), \quad r(0) \geq 1
$$

and the fact that, for $r>0, \frac{r-1}{r} \leq 1$, yields $\dot{V}_{1} \leq-\frac{k}{4}|\boldsymbol{\eta}|^{2}$. Thus $\boldsymbol{\eta}=\mathbf{0}$ is GES. Note that $\mathbf{z}=0$ will be GES if it can be proved that $r \in \mathcal{L}_{\infty}$. Before going through this proof, let us show that $\mathbf{e}_{\mathbf{q}}=\mathbf{0}$ and $\mathbf{e}_{\mathbf{x}}=\mathbf{0}$ are also GES. First, let us start by setting

$$
\begin{aligned}
\dot{\hat{\mathbf{q}}} & =\mathbf{L}(\mathbf{q})(\boldsymbol{\xi}+\boldsymbol{\beta})-\psi_{2}(\mathbf{q}, r) \mathbf{e}_{\mathbf{q}} \\
\hat{\dot{\mathbf{x}}} & =\mathbf{F}(\mathbf{q}, \boldsymbol{\tau}, \mathbf{w})+\mathbf{S}(\mathbf{q}, \boldsymbol{\xi}+\boldsymbol{\beta})(\boldsymbol{\xi}+\boldsymbol{\beta})-\psi_{1}(\mathbf{q}, r) \mathbf{e}_{\mathbf{x}},
\end{aligned}
$$

where $\psi_{1}, \psi_{2}: \mathbb{R}^{2 n} \times \mathbb{R} \rightarrow \mathbb{R}_{>0}$ will be defined later. Using the previous expressions, (13) can be written as

$$
\begin{aligned}
& \dot{\mathbf{e}}_{\mathbf{x}}=\left(\nabla_{\mathbf{q}} \boldsymbol{\beta}\right) \mathbf{L}(\mathbf{q}) r \boldsymbol{\eta}-\psi_{1}(\mathbf{q}, r) \mathbf{e}_{\mathbf{x}} \\
& \dot{\mathbf{e}}_{\mathbf{q}}=\mathbf{L}(\mathbf{q}) r \boldsymbol{\eta}-\psi_{2}(\mathbf{q}, r) \mathbf{e}_{\mathbf{q}} .
\end{aligned}
$$

Now, let us define the proper Lyapunov candidate function

$$
V_{2}=V_{1}+\frac{1}{2}\left(\left|\mathbf{e}_{\mathbf{x}}\right|^{2}+\left|\mathbf{e}_{\mathbf{q}}\right|^{2}\right) .
$$

After applying Young's inequality, $\dot{V}_{2}$ evaluated along (18), yields

$$
\dot{V}_{2} \leq-\left(\frac{k}{4}-1\right)|\boldsymbol{\eta}|^{2}-\left(\psi_{1}-\left.\frac{1}{2} r^{2}\left\|\nabla_{\mathbf{q}} \boldsymbol{\beta}\right\|\right|^{2}\|\mathbf{L}\|^{2}\right)\left|\mathbf{e}_{\mathbf{x}}\right|^{2}-\left(\psi_{2}-\frac{1}{2} r^{2}|| \mathbf{L} \|^{2}\right)\left|\mathbf{e}_{\mathbf{q}}\right|^{2}
$$

Clearly, if we set $k:=4\left(1+\psi_{3}\right), \psi_{1}:=\frac{1}{2} r^{2}\left\|\nabla_{\mathbf{q}} \boldsymbol{\beta}\right\|\left\|^{2}\right\| \mathbf{L} \|^{2}+\psi_{4}$ and $\psi_{2}:=\frac{1}{2} r^{2}\|\mathbf{L}\|^{2}+\psi_{5}$, where $\psi_{3}, \psi_{4}, \psi_{5} \in \mathbb{R}_{>0}$ and which will be explicitly defined in the following section, then $\dot{V}_{2} \leq$ $-\psi_{3}|\boldsymbol{\eta}|^{2}-\psi_{4}\left|\mathbf{e}_{\mathbf{x}}\right|^{2}-\psi_{5}\left|\mathbf{e}_{\mathbf{q}}\right|^{2}$. Hence, $\mathbf{e}_{\mathbf{x}}=\mathbf{0}$ and $\mathbf{e}_{\mathbf{q}}=\mathbf{0}$ are also GES.

Summarizing, the complete observer dynamics is given by

$$
\begin{aligned}
\dot{\boldsymbol{\xi}} & =\mathbf{F}(\mathbf{q}, \boldsymbol{\tau}, \mathbf{w})-\nabla_{\hat{\mathbf{q}}} \boldsymbol{\beta} \dot{\hat{\mathbf{q}}}-\nabla_{\hat{\mathbf{x}}} \boldsymbol{\beta} \dot{\hat{\mathbf{x}}}+\mathbf{S}(\mathbf{q}, \boldsymbol{\xi}+\boldsymbol{\beta})(\boldsymbol{\xi}+\boldsymbol{\beta})-\nabla_{\mathbf{q}} \boldsymbol{\beta} \mathbf{L}(\mathbf{q})(\boldsymbol{\xi}+\boldsymbol{\beta}) \\
\dot{r} & =-\frac{k}{4}(r-1)+\frac{r}{k}\left(\left\|\boldsymbol{\Delta}_{\mathbf{x}} \mathbf{L}\right\|^{2}+\left\|\boldsymbol{\Delta}_{\mathbf{q}} \mathbf{L}\right\|^{2}\right), \quad r(0) \geq 1 \\
\dot{\hat{\mathbf{q}}} & =\mathbf{L}(\mathbf{q})(\boldsymbol{\xi}+\boldsymbol{\beta})-\frac{1}{2} r^{2}\|\mathbf{L}\|^{2} \mathbf{e}_{\mathbf{q}}-\psi_{5} \mathbf{e}_{\mathbf{q}} \\
\hat{\dot{\mathbf{x}}} & =\mathbf{F}(\mathbf{q}, \boldsymbol{\tau}, \mathbf{w})+\mathbf{S}(\mathbf{q}, \boldsymbol{\xi}+\boldsymbol{\beta})(\boldsymbol{\xi}+\boldsymbol{\beta})-\frac{1}{2} r^{2}\left\|\nabla_{\mathbf{q}} \boldsymbol{\beta}\right\|^{2}\|\mathbf{L}\|^{2} \mathbf{e}_{\mathbf{x}}-\psi_{4} \mathbf{e}_{\mathbf{x}}
\end{aligned}
$$

It remains to prove that $r \in \mathcal{L}_{\infty}$. This last, together with the stability of the teleoperator controlled by $\mathrm{P}+\mathrm{d}$ controllers, with and without time-delays, and in closed loop with the velocity observer, is shown in the following section. 


\section{POSITION TRACKING FOR TELEOPERATORS WITHOUT VELOCITY MEASUREMENTS}

Suppose that the velocities $\dot{\mathbf{q}}=\mathbf{L}(\mathbf{q}) \mathbf{x}$ are not available for measurement. The objective of this section is to prove, for the undelayed and the delayed cases, that the incorporation in the P+d controllers of the estimated velocities $\hat{\dot{\mathbf{q}}}=\mathbf{L}(\mathbf{q}) \hat{\mathbf{x}}$, provided by the aforementioned I\&I observer, ensures global boundedness of the closed-loop trajectories and convergence of velocities to zero, as well as position tracking in the absence of interaction forces. A general scheme for observer-based teleoperation control is depicted in Figure 1.

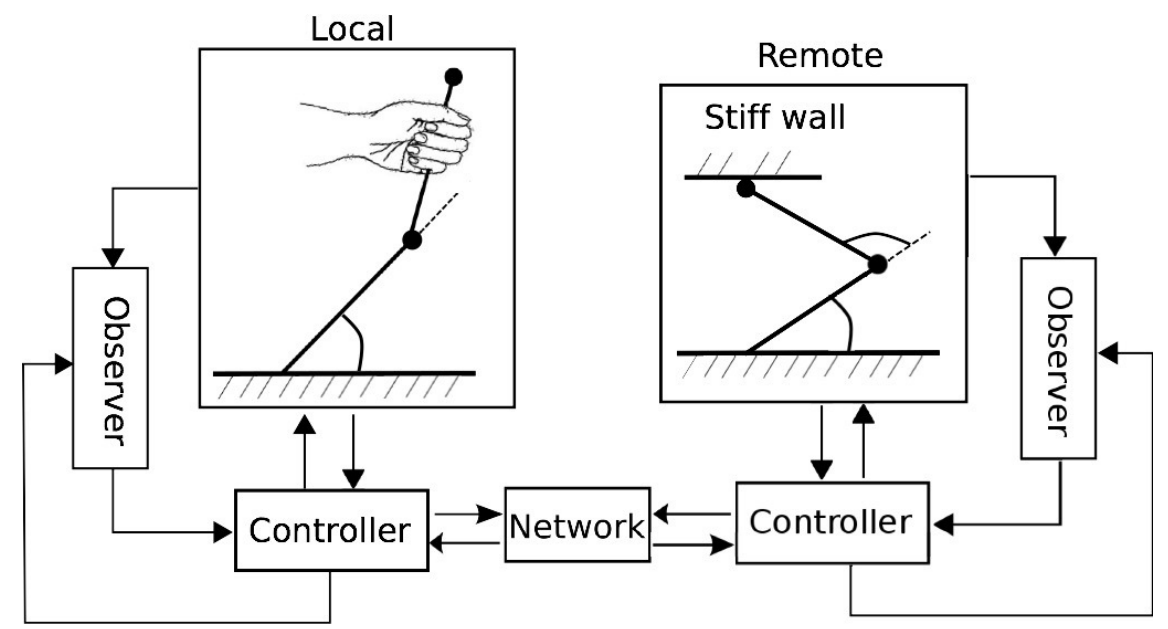

Figure 1. Observer-based teleoperation control scheme.

First, the stability of the bilateral teleoperator in closed-loop with the P+d controllers

$$
\begin{aligned}
& \boldsymbol{\tau}_{l}=K_{l}\left[\mathbf{q}_{l}-\mathbf{q}_{r}\right]+B_{l} \mathbf{L}_{l} \hat{\mathbf{x}}_{l}-\mathbf{g}_{l}\left(\mathbf{q}_{l}\right) \\
& \boldsymbol{\tau}_{r}=-K_{r}\left[\mathbf{q}_{r}-\mathbf{q}_{l}\right]-B_{r} \mathbf{L}_{r} \hat{\mathbf{x}}_{r}+\mathbf{g}_{r}\left(\mathbf{q}_{r}\right),
\end{aligned}
$$

is analized for the undelayed case. Then, the case where variable time-delays arise in the communications follows. In this scenario, the controllers (21) change to

$$
\begin{aligned}
& \boldsymbol{\tau}_{l}=K_{l}\left[\mathbf{q}_{l}-\mathbf{q}_{r}\left(t-T_{r}(t)\right)\right]+B_{l} \mathbf{L}_{l} \hat{\mathbf{x}}_{l}-\mathbf{g}_{l}\left(\mathbf{q}_{l}\right) \\
& \boldsymbol{\tau}_{r}=-K_{r}\left[\mathbf{q}_{r}-\mathbf{q}_{l}\left(t-T_{l}(t)\right)\right]-B_{r} \mathbf{L}_{r} \hat{\mathbf{x}}_{r}+\mathbf{g}_{r}\left(\mathbf{q}_{r}\right) .
\end{aligned}
$$

In both cases, $K_{i}, B_{i} \in \mathbb{R}_{>0}$ are control gains.

\subsection{The Undelayed Case}

To this end, note that (21) can be compactly written as

$$
\boldsymbol{\tau}=-\mathbf{K q}-\mathbf{B L}(\mathbf{q}) \mathbf{x}+\mathbf{g}(\mathbf{q})-\mathbf{B L}(\hat{\mathbf{x}}-\mathbf{x})
$$


with the constant matrices $\mathbf{K}, \mathbf{B} \in \mathbb{R}^{2 n \times 2 n}$ defined as

$$
\mathbf{K}:=\left[\begin{array}{cc}
K_{l} \mathbf{I} & -K_{l} \mathbf{I} \\
-K_{r} \mathbf{I} & K_{r} \mathbf{I}
\end{array}\right], \quad \mathbf{B}:=\left[\begin{array}{cc}
B_{l} \mathbf{I} & \varnothing \\
\varnothing & B_{r} \mathbf{I}
\end{array}\right]
$$

Replacing (23) in (5) and using (3), yields the following closed-loop dynamics

$$
\begin{aligned}
\dot{\mathbf{q}} & =\mathbf{L}(\mathbf{q}) \mathbf{x} \\
\dot{\mathbf{x}} & =\mathbf{S}(\mathbf{q}, \mathbf{x}) \mathbf{x}+\mathbf{L}^{\top}(\mathbf{q})(\mathbf{w}-\mathbf{K} \mathbf{q}-\mathbf{B L}(\mathbf{q}) \mathbf{x})-\mathbf{L}^{\top}(\mathbf{q}) \mathbf{B L}(\mathbf{q})(\hat{\mathbf{x}}-\mathbf{x})
\end{aligned}
$$

Moreover, from the observer design we have that

$$
\left.\begin{array}{rl}
\mathbf{z} & =\boldsymbol{\xi}+\boldsymbol{\beta}-\mathbf{x} \\
\mathbf{e}_{\mathbf{x}} & =\hat{\mathbf{x}}-(\boldsymbol{\xi}+\boldsymbol{\beta}) \\
\mathbf{z} & =r \boldsymbol{\eta}
\end{array}\right\} \Rightarrow \hat{\mathbf{x}}-\mathbf{x}=r \boldsymbol{\eta}+\mathbf{e}_{\mathbf{x}}
$$

Thus, by using $\hat{\mathbf{x}}-\mathbf{x}=r \boldsymbol{\eta}+\mathbf{e}_{\mathbf{x}}$, the closed-loop dynamics can further be written as

$$
\begin{aligned}
\dot{\mathbf{q}} & =\mathbf{L}(\mathbf{q}) \mathbf{x} \\
\dot{\mathbf{x}} & =\mathbf{S}(\mathbf{q}, \mathbf{x}) \mathbf{x}+\mathbf{L}^{\top}(\mathbf{q})(\mathbf{w}-\mathbf{K} \mathbf{q}-\mathbf{B L}(\mathbf{q}) \mathbf{x})-\mathbf{L}^{\top}(\mathbf{q}) \mathbf{B} \mathbf{L}(\mathbf{q})\left(r \boldsymbol{\eta}+\mathbf{e}_{\mathbf{x}}\right) .
\end{aligned}
$$

Note that the complete description of the system is composed of a twofold, that is, the observer dynamics (20) plus the local and remote closed-loop equations (24).

The following proposition states one of the results of the present work.

\section{Proposition 1}

Consider the closed-loop teleoperator system (24). If Assumptions A1-A2 are satisfied, then for any $K_{i}, B_{i} \in \mathbb{R}_{>0}$, it holds that

(i) Velocity and position error are globally bounded, i.e. $\dot{\mathbf{q}},\left|\mathbf{q}_{l}-\mathbf{q}_{r}\right| \in \mathcal{L}_{\infty}$ and $\dot{\mathbf{q}} \in \mathcal{L}_{2}$.

(ii) Moreover, if the human and environment forces are zero, i.e. $\boldsymbol{\tau}_{h}=\boldsymbol{\tau}_{e} \equiv \mathbf{0}$, then velocity and position error exhibit global asymptotic convergence to zero, i.e.

$$
\lim _{t \rightarrow \infty}|\dot{\mathbf{q}}|=0, \quad \lim _{t \rightarrow \infty}\left|\mathbf{q}_{l}-\mathbf{q}_{r}\right|=0
$$

Proof

Consider the following Lyapunov-like function

$$
V\left(\mathbf{x}, \mathbf{q}, \boldsymbol{\eta}, \mathbf{e}_{\mathbf{x}}, \mathbf{e}_{\mathbf{q}}, r, t\right)=V_{2}\left(\boldsymbol{\eta}, \mathbf{e}_{\mathbf{x}}, \mathbf{e}_{\mathbf{q}}\right)+V_{3}(\mathbf{x}, \mathbf{q}, t)+\frac{1}{2} r^{2},
$$

where $V_{2}$ has been defined in (19) and

$$
V_{3}(\mathbf{x}, \mathbf{q}, t):=\frac{1}{2} \mathbf{x}^{\top} \mathbf{\Sigma} \mathbf{x}+\frac{1}{2} \mathbf{q}^{\top} \tilde{\mathbf{K}} \mathbf{q}+E_{h}+E_{e}
$$

with $\boldsymbol{\Sigma}:=\operatorname{diag}\left(\mathbf{I}, \frac{K_{l}}{K_{r}} \mathbf{I}\right), \tilde{\mathbf{K}}:=\boldsymbol{\Sigma} \mathbf{K}$ and $E_{h}, E_{e}$ given by (2). Since $K_{i} \in \mathbb{R}_{>0}, \boldsymbol{\Sigma}$ is diagonal and positive definite, and $\tilde{\mathbf{K}}=K_{l}\left[\begin{array}{cc}\mathbf{I} & -\mathbf{I} \\ -\mathbf{I} & \mathbf{I}\end{array}\right]$ is positive semi-definite, thus $V$ is positive semi-definite 
and radially unbounded w.r.t. $\left|\mathbf{q}_{l}-\mathbf{q}_{r}\right|, \mathbf{x}, \boldsymbol{\eta}, \mathbf{e}_{\mathbf{x}}, \mathbf{e}_{\mathbf{q}}$ and $r$. Its time derivative along trajectories (16), (18) and (24) meets

$$
\dot{V} \leq-\mathbf{x}^{\top} \mathbf{L}^{\top} \tilde{\mathbf{B}} \mathbf{L} \mathbf{x}-\mathbf{x}^{\top} \mathbf{L}^{\top} \tilde{\mathbf{B}} \mathbf{L}\left(r \boldsymbol{\eta}+\mathbf{e}_{\mathbf{x}}\right)-\psi_{3}|\boldsymbol{\eta}|^{2}-\psi_{4}\left|\mathbf{e}_{\mathbf{x}}\right|^{2}-\psi_{5}\left|\mathbf{e}_{\mathbf{q}}\right|^{2}+r \dot{r}
$$

where $\tilde{\mathbf{B}}=\boldsymbol{\Sigma} \mathbf{B}$ and $\mathbf{K}_{h}=\mathbf{I}, \mathbf{K}_{e}=\frac{K_{l}}{K_{r}} \mathbf{I}$. Since the matrix $\tilde{\mathbf{B}}$ is diagonal and positive definite we have that

$$
-\mathbf{x}^{\top} \mathbf{L}^{\top}(\mathbf{q}) \tilde{\mathbf{B}} \mathbf{L}(\mathbf{q}) \mathbf{x} \leq-b|\mathbf{L}(\mathbf{q}) \mathbf{x}|^{2},
$$

where $b:=\min \left\{B_{l}, \frac{B_{r} K_{l}}{K_{r}}\right\}$. Using Young's inequality, it can be written for any $\alpha \in \mathbb{R}_{>0},{ }^{\dagger}$

$$
\begin{aligned}
-\mathbf{x}^{\top} \mathbf{L}^{\top} \tilde{\mathbf{B}} \mathbf{L}\left(r \boldsymbol{\eta}+\mathbf{e}_{\mathbf{x}}\right) & \leq \frac{\alpha}{2}|\tilde{\mathbf{B}} \mathbf{L} \mathbf{x}|^{2}+\frac{1}{2 \alpha}\left|r \mathbf{L} \boldsymbol{\eta}+\mathbf{L} \mathbf{e}_{\mathbf{x}}\right|^{2} \\
& \leq \frac{\alpha B^{2}}{2}|\mathbf{L} \mathbf{x}|^{2}+\frac{1}{\alpha} r^{2}\|\mathbf{L}\|^{2}|\boldsymbol{\eta}|^{2}+\frac{1}{\alpha}\|\mathbf{L}\|^{2}\left|\mathbf{e}_{\mathbf{x}}\right|^{2},
\end{aligned}
$$

where $B:=\max \left\{B_{l}, \frac{B_{r} K_{l}}{K_{r}}\right\}$. Substituting (17) in (27), with the fact that, for $r>0, \frac{r-1}{r} \leq 1$, and the previous bounds, with $\alpha=\frac{b}{B^{2}}$, yields

$$
\begin{aligned}
\dot{V} \leq & -\frac{b}{2}|\mathbf{L x}|^{2}-\left(\psi_{3}-\frac{B^{2}}{b} r^{2}|| \mathbf{L} \|^{2}\right)|\boldsymbol{\eta}|^{2}-\left(\psi_{4}-\frac{B^{2}}{b}\|\mathbf{L}\|^{2}\right)\left|\mathbf{e}_{\mathbf{x}}\right|^{2}-\psi_{5}\left|\mathbf{e}_{\mathbf{q}}\right|^{2}+ \\
& +\frac{r^{2}}{4\left(1+\psi_{3}\right)}\left(\left\|\boldsymbol{\Delta}_{\mathbf{x}} \mathbf{L}\right\|^{2}+\left\|\mathbf{\Delta}_{\mathbf{q}} \mathbf{L}\right\|^{2}\right) .
\end{aligned}
$$

Now, (14) ensures the existence of mappings $\bar{\Delta}_{\mathbf{q}}, \bar{\Delta}_{\mathbf{x}}: \mathbb{R}^{2 n} \times \mathbb{R}^{2 n} \times \mathbb{R}^{2 n} \rightarrow \mathbb{R}^{2 n \times 2 n}$ such that

$$
\begin{aligned}
\left\|\boldsymbol{\Delta}_{\mathbf{q}}\left(\mathbf{q}, \hat{\mathbf{x}}, \mathbf{e}_{\mathbf{q}}\right)\right\| & \leq\left\|\bar{\Delta}_{\mathbf{q}}\left(\mathbf{q}, \hat{\mathbf{x}}, \mathbf{e}_{\mathbf{q}}\right)\right\|\left|\mathbf{e}_{\mathbf{q}}\right| \\
\left\|\boldsymbol{\Delta}_{\mathbf{x}}\left(\mathbf{q}, \hat{\mathbf{x}}, \mathbf{e}_{\mathbf{x}}\right)\right\| & \leq\left\|\bar{\Delta}_{\mathbf{x}}\left(\mathbf{q}, \hat{\mathbf{x}}, \mathbf{e}_{\mathbf{x}}\right)\right\|\left|\mathbf{e}_{\mathbf{x}}\right|
\end{aligned}
$$

These bounds ensure that

$$
\left\|\boldsymbol{\Delta}_{\mathbf{x}} \mathbf{L}\right\|^{2}+\left\|\boldsymbol{\Delta}_{\mathbf{q}} \mathbf{L}||^{2} \leq\right\| \mathbf{L}\left\|^{2}|| \overline{\boldsymbol{\Delta}}_{\mathbf{x}}||^{2}\left|\mathbf{e}_{\mathbf{x}}\right|^{2}+\right\| \mathbf{L}\left\|^{2}\right\| \overline{\boldsymbol{\Delta}}_{\mathbf{q}}||^{2}\left|\mathbf{e}_{\mathbf{q}}\right|^{2} .
$$

Finally, setting

$$
\begin{aligned}
\psi_{3} & =\frac{B^{2}}{b} r^{2}\|\mathbf{L}(\mathbf{q})\|^{2}+c_{1} \\
\psi_{4} & =\frac{B^{2}}{b}\|\mathbf{L}(\mathbf{q})\|^{2}+\frac{r^{2}}{4\left(1+\psi_{3}\right)}\|\mathbf{L}(\mathbf{q})\|^{2}\left\|\overline{\boldsymbol{\Delta}}_{\mathbf{x}}\left(\mathbf{q}, \hat{\mathbf{x}}, \mathbf{e}_{\mathbf{x}}\right)\right\|^{2}+c_{2} \\
\psi_{5} & =\frac{r^{2}}{4\left(1+\psi_{3}\right)}\|\mathbf{L}(\mathbf{q})\|^{2}\left\|\overline{\boldsymbol{\Delta}}_{\mathbf{q}}\left(\mathbf{q}, \hat{\mathbf{x}}, \mathbf{e}_{\mathbf{q}}\right)\right\|^{2}+c_{3},
\end{aligned}
$$

for any $c_{1}, c_{2}, c_{3} \in \mathbb{R}_{>0}$, yields

$$
\dot{V} \leq-\frac{b}{2}|\mathbf{L}(\mathbf{q}) \mathbf{x}|^{2}-c_{1}|\boldsymbol{\eta}|^{2}-c_{2}\left|\mathbf{e}_{\mathbf{x}}\right|^{2}-c_{3}\left|\mathbf{e}_{\mathbf{q}}\right|^{2} .
$$

†The fact that $(a+b)^{2} \leq 2\left(a^{2}+b^{2}\right)$ is also used. 
The fact that $V \geq 0$ and $\dot{V} \leq 0$, and that $\mathbf{L}$ is full rank, ensures that $\mathbf{x}, \boldsymbol{\eta}, \mathbf{e}_{\mathbf{x}}, \mathbf{e}_{\mathbf{q}} \in \mathcal{L}_{\infty} \cap \mathcal{L}_{2}$ and that $\left|\mathbf{q}_{l}-\mathbf{q}_{r}\right|, r \in \mathcal{L}_{\infty}$. This completes part (i) of the proof.

Since $r \in \mathcal{L}_{\infty}$ and $\boldsymbol{\eta}=0$ is GES, it is proved that $\mathbf{z}=\mathbf{0}$ is also GES.

In the case when $\boldsymbol{\tau}_{h}=\boldsymbol{\tau}_{e} \equiv \mathbf{0}$, we have that $\mathrm{x} \in \mathcal{L}_{\infty} \cap \mathcal{L}_{2}$ and $\boldsymbol{\tau} \in \mathcal{L}_{\infty}$ which, together with (24) and Assumption A1, prove that $\dot{\mathbf{x}} \in \mathcal{L}_{\infty}$. Hence, from Barbalăt's Lemma, $\lim _{t \rightarrow \infty}|\mathbf{x}(t)| \rightarrow 0$.

Additionally, from (24) and the fact that $\boldsymbol{\eta}=0, \mathbf{e}_{\mathbf{x}}=0$ are GES, the claim that $\lim _{t \rightarrow \infty} \mid \mathbf{q}_{l}(t)-$ $\mathbf{q}_{r}(t) \mid=0$ is established if $\dot{\mathbf{x}}$ asymptotically converges to zero. For, it suffices to prove that $\dot{\mathbf{x}}$ is uniformly continuous because $\lim _{t \rightarrow \infty} \int_{0}^{t} \dot{\mathbf{x}}(\sigma) d \sigma=-\mathbf{x}(0)$ due to $|\mathbf{x}(t)| \rightarrow 0$. Now, from (24) together with Property P5, it can be shown that $\ddot{x}$ is a function of bounded signals, hence $\ddot{x} \in \mathcal{L}_{\infty}$. This last fact ensures that $\dot{x}$ is uniformly continuous, as required. This completes the proof.

Hence, referring to the initial coordinates it is concluded that $\Psi^{-1}(\mathbf{q}) \hat{\mathbf{x}}$ is an asymptotic estimate of $\dot{\mathbf{q}}$.

\section{Remark 1}

One can note two differences with respect to the original I\&I observer proposed in [22], [23]. The first one is that now the mapping $\mathbf{F}(\mathbf{q}, \boldsymbol{\tau}, \mathbf{w})$ also contains the (time-varying) interaction forces with the human and the environment. Thus, the stability proof requires different arguments than those reported in [22,23]. The second difference is that $k_{1}$ is not a positive constant but rather a positive function of $r$. This modification does not alter the properties of the observer neither the $r$-dynamics and, in fact, as it is shown in this section, it is used to dominate the cross-terms appearing from the observer-plant-controller interconnection.

\section{Remark 2}

From (3) and the observer dynamics (20), it should be noted that, for the observer implementation, the human operator and the environment forces have to be measurable. If these are not measured or there is uncertainty in such measurements, then these forces can be treated as disturbances and Input-to-State Stability can be concluded. Similar to the work reported in [30].

\subsection{The Delayed Case}

In the delayed case, the teleoperator dynamics (24) transforms to

$$
\begin{aligned}
\dot{\mathbf{q}} & =\mathbf{L}(\mathbf{q}) \mathbf{x} \\
\dot{\mathbf{x}} & =\mathbf{S}(\mathbf{q}, \mathbf{x}) \mathbf{x}+\mathbf{L}^{\top}\left(\mathbf{w}-\mathbf{K q}-\left[\begin{array}{c}
K_{l} \int_{-T_{r}(t)}^{0} \dot{\mathbf{q}}_{r}(t+\theta) d \theta \\
K_{r} \int_{-T_{l}(t)}^{0} \dot{\mathbf{q}}_{l}(t+\theta) d \theta
\end{array}\right]-\mathbf{B L x}\right)-\mathbf{L}^{\top} \mathbf{B L}\left(r \boldsymbol{\eta}+\mathbf{e}_{\mathbf{x}}\right),
\end{aligned}
$$

where the fact that $\int_{-T_{i}(t)}^{0} \dot{\mathbf{q}}_{i}(t+\theta) d \theta=\mathbf{q}_{i}(t)-\mathbf{q}_{i}\left(t-T_{i}(t)\right)$ is used.

The main result of this work is the following:

Proposition 2

Consider the closed-loop teleoperator (29). Suppose that Assumptions A1-A3 hold. Then, if the controller gains are set fulfilling

$$
B_{l} B_{r}>\left({ }^{*} T_{l}+{ }^{*} T_{r}\right)^{2} K_{l} K_{r},
$$


the following holds:

(i) Velocity and position error are globally bounded, i.e. $\dot{\mathbf{q}},\left|\mathbf{q}_{l}-\mathbf{q}_{r}\right| \in \mathcal{L}_{\infty}$ and $\dot{\mathbf{q}} \in \mathcal{L}_{2}$.

(ii) Moreover, if the human and environment forces are zero, i.e. $\boldsymbol{\tau}_{h}=\boldsymbol{\tau}_{e} \equiv \mathbf{0}$, then velocity and position error exhibit global asymptotic convergence to zero, i.e.

$$
\lim _{t \rightarrow \infty}|\dot{\mathbf{q}}|=0, \quad \lim _{t \rightarrow \infty}\left|\mathbf{q}_{l}-\mathbf{q}_{r}\right|=0
$$

Proof

Consider $V_{3}(\mathbf{x}, \mathbf{q}, t)$, defined in (26), and evaluate its time-derivative along (29). This yields,

$$
\dot{V}_{3}=-\mathbf{x}^{\top} \mathbf{L}^{\top} \tilde{\mathbf{B}} \mathbf{L} \mathbf{x}-K_{l} \mathbf{x}^{\top} \mathbf{L}^{\top}\left[\begin{array}{c}
\int_{-T_{r}(t)}^{0} \dot{\mathbf{q}}_{r}(t+\theta) d \theta \\
\int_{-T_{l}(t)}^{0} \dot{\mathbf{q}}_{l}(t+\theta) d \theta
\end{array}\right]-\mathbf{x}^{\top} \mathbf{L}^{\top} \tilde{\mathbf{B}} \mathbf{L}\left(r \boldsymbol{\eta}+\mathbf{e}_{\mathbf{x}}\right) .
$$

Proceeding as in the previous proof, using the positive semi-definite function $V$ in (25) and evaluating $\dot{V}$ along (16), (17), (18) and (29) with

$$
\begin{aligned}
\psi_{3} & =\frac{B^{2}}{b} r^{2}\|\mathbf{L}(\mathbf{q})\|^{2}+c_{1} \\
\psi_{4} & =\frac{B^{2}}{b}\|\mathbf{L}(\mathbf{q})\|^{2}+\frac{r^{2}}{4\left(1+\psi_{3}\right)}\|\mathbf{L}(\mathbf{q})\|^{2}\left\|\overline{\boldsymbol{\Delta}}_{\mathbf{x}}\left(\mathbf{q}, \hat{\mathbf{x}}, \mathbf{e}_{\mathbf{x}}\right)\right\|^{2}+c_{2} \\
\psi_{5} & =\frac{r^{2}}{4\left(1+\psi_{3}\right)}\|\mathbf{L}(\mathbf{q})\|^{2}\left\|\overline{\boldsymbol{\Delta}}_{\mathbf{q}}\left(\mathbf{q}, \hat{\mathbf{x}}, \mathbf{e}_{\mathbf{q}}\right)\right\|^{2}+c_{3},
\end{aligned}
$$

yields (after applying Young's inequality and the fact that $\dot{\mathbf{q}}=\mathbf{L x}$ )

$$
\dot{V} \leq-\frac{1}{2} \dot{\mathbf{q}}^{\top} \tilde{\mathbf{B}} \dot{\mathbf{q}}-K_{l} \dot{\mathbf{q}}^{\top}\left[\begin{array}{c}
\int_{-T_{r}(t)}^{0} \dot{\mathbf{q}}_{r}(t+\theta) d \theta \\
\int_{-T_{l}(t)}^{0} \dot{\mathbf{q}}_{l}(t+\theta) d \theta
\end{array}\right]-c_{1}|\boldsymbol{\eta}|^{2}-c_{2}\left|\mathbf{e}_{\mathbf{x}}\right|^{2}-c_{3}\left|\mathbf{e}_{\mathbf{q}}\right|^{2},
$$

where $c_{1}, c_{2}, c_{3} \in \mathbb{R}_{>0}$. Defining $\chi:=\operatorname{col}\left(\boldsymbol{\eta}, \mathbf{e}_{\mathbf{x}}, \mathbf{e}_{\mathbf{q}}\right)$ and $c_{m}:=\min \left\{c_{1}, c_{2}, c_{3}\right\}$, the previous inequality can be further written as

$$
\dot{V} \leq-\frac{B_{l}}{2}\left|\dot{\mathbf{q}}_{l}\right|^{2}-\frac{B_{r} K_{l}}{2 K_{r}}\left|\dot{\mathbf{q}}_{r}\right|^{2}-K_{l} \dot{\mathbf{q}}_{l}^{\top} \int_{-T_{r}(t)}^{0} \dot{\mathbf{q}}_{r}(t+\theta) d \theta-K_{l} \dot{\mathbf{q}}_{r}^{\top} \int_{-T_{l}(t)}^{0} \dot{\mathbf{q}}_{l}(t+\theta) d \theta-c_{m}|\chi|^{2} .
$$

Now, integrating $\dot{V}$, from 0 to $t$, yields

$$
\begin{aligned}
V(t)-V(0) \leq & -c_{m}\|\chi\|_{2}^{2}-\frac{B_{l}}{2}\left\|\dot{\mathbf{q}}_{l}\right\|_{2}^{2}-\frac{B_{r} K_{l}}{2 K_{r}}\left\|\dot{\mathbf{q}}_{r}\right\|_{2}^{2}- \\
& -K_{l} \int_{0}^{t} \dot{\mathbf{q}}_{l}^{\top}(\sigma) \int_{-T_{r}(\sigma)}^{0} \dot{\mathbf{q}}_{r}(\sigma+\theta) d \theta d \sigma-K_{l} \int_{0}^{t} \dot{\mathbf{q}}_{r}^{\top}(\sigma) \int_{-T_{l}(\sigma)}^{0} \dot{\mathbf{q}}_{l}(\sigma+\theta) d \theta d \sigma .
\end{aligned}
$$

Invoking Lemma 1 to the double integral terms, with $\alpha_{l}$ and $\alpha_{r}$, respectively, yields

$$
V(t)-V(0) \leq-c_{m}\|\chi\|_{2}^{2}-\lambda_{l}\left\|\dot{\mathbf{q}}_{l}\right\|_{2}^{2}-\lambda_{r}\left\|\dot{\mathbf{q}}_{r}\right\|_{2}^{2}
$$

where $\lambda_{l}:=B_{l}-\alpha_{l} K_{l}-\frac{{ }^{*} T_{l}^{2} K_{l}}{\alpha_{r}}$ and $\lambda_{r}:=B_{r}-\alpha_{r} K_{r}-\frac{{ }^{*} T_{r}^{2} K_{r}}{\alpha_{l}}$. Note that $V(t) \geq 0$ and if $\lambda_{i}>0$ then $\chi, \dot{\mathbf{q}} \in \mathcal{L}_{2}$. It can be easily shown that there is a solution for $\lambda_{i}>0$ and $\alpha_{i}>0$ if $B_{l} B_{r}>$ 
$\left({ }^{*} T_{l}+{ }^{*} T_{r}\right)^{2} K_{l} K_{r}$. Thus, setting the control gains such that (30) holds, ensures that $\chi, \dot{\mathbf{q}} \in \mathcal{L}_{2}$ and, moreover, that $V(t) \leq V(0)$. This last fact, together with the fact that $V(t)$ is radially unbounded with regards to $\mathbf{q}_{l}-\mathbf{q}_{r}, \mathbf{x}, \boldsymbol{\eta}, \mathbf{e}_{\mathbf{x}}, \mathbf{e}_{\mathbf{q}}$ and $r$, proves that $\mathbf{q}_{l}-\mathbf{q}_{r}, \mathbf{x}, \boldsymbol{\eta}, \mathbf{e}_{\mathbf{x}}, \mathbf{e}_{\mathbf{q}}, r \in \mathcal{L}_{\infty}$. This completes the proof of Part (i).

The proof of the last part follows verbatim the proof of part (ii) of Proposition 1 with the following additional facts: i) $\int_{-T_{i}(t)}^{0}\left|\dot{\mathbf{q}}_{i}(t+\theta)\right| d \theta \leq{ }^{*} T_{i}^{\frac{1}{2}}\left\|\dot{\mathbf{q}}_{i}\right\|_{2}<\infty$ (using Schwartz inequality) and ii) if $\lim _{t \rightarrow \infty} \dot{\mathbf{q}}_{i}(t)=\mathbf{0}$ then $\lim _{t \rightarrow \infty} \int_{-T_{i}(t)}^{0} \dot{\mathbf{q}}_{i}(t+\theta) d \theta=\mathbf{0}$.

\section{SIMULATIONS}

To show the effectiveness of the proposed scheme, this section presents some simulations with and without interaction with the remote environment. The local and remote manipulators are modeled as a pair of 2-DOF serial links with revolute joints. The simulation platform follows Figure 1. The corresponding nonlinear dynamics are modeled using (1) with the inertia and Coriolis matrices given by

$$
\mathbf{M}_{i}\left(\mathbf{q}_{i}\right)=\left[\begin{array}{cc}
\alpha_{i}+2 \beta_{i} \mathrm{c}_{2_{i}} & \delta_{i}+\beta_{i} \mathrm{c}_{2_{i}} \\
\delta_{i}+\beta_{i} \mathrm{c}_{2_{i}} & \delta_{i}
\end{array}\right], \quad \mathbf{C}_{i}\left(\mathbf{q}_{i}, \dot{\mathbf{q}}_{i}\right)=\left[\begin{array}{cc}
-\beta_{i} \mathrm{~s}_{2_{i}} \dot{q}_{2_{i}} & -\beta_{i} \mathrm{~s}_{2_{i}}\left(\dot{q}_{1_{i}}+\dot{q}_{2_{i}}\right) \\
\beta_{i} \mathrm{~s}_{2_{i}} \dot{q}_{1_{i}} & 0
\end{array}\right]
$$

and the gravity vector given by

$$
\mathbf{g}_{i}\left(\mathbf{q}_{i}\right)=\left[\begin{array}{c}
\frac{1}{l_{2_{i}}} g \delta_{i} \mathrm{c}_{12_{i}}+\frac{1}{l_{1_{i}}}\left(\alpha_{i}-\delta_{i}\right) \mathrm{c}_{1_{i}} \\
\frac{1}{l_{2_{i}}} g \delta_{i} \mathrm{c}_{12_{i}}
\end{array}\right],
$$

where $\alpha_{i}:=l_{2_{i}}^{2} m_{2_{i}}+l_{1_{i}}^{2}\left(m_{1_{i}}+m_{2_{i}}\right), \beta_{i}:=l_{1_{i}} l_{2_{i}} m_{2_{i}}$ and $\delta_{i}:=l_{2_{i}}^{2} m_{2_{i}}$.

$\mathrm{c}_{2_{i}}, \mathrm{~s}_{2_{i}}$ and $\mathrm{c}_{12_{i}}$ stand for the short notation of $\cos \left(q_{2_{i}}\right), \sin \left(q_{2_{i}}\right)$ and $\cos \left(q_{1_{i}}+q_{2_{i}}\right)$, respectively. $q_{k_{i}}$ and $\dot{q}_{k_{i}}$ are the joint position and velocity, respectively, of link $k$ of manipulator $i$, with $k \in\{1,2\} . l_{k_{i}}$ and $m_{k_{i}}$ are the respective lengths and masses of each link.

The mapping $\boldsymbol{\Psi}_{i}\left(\mathbf{q}_{i}\right)$, found using the Cholesky factorization, and its inverse $\mathbf{L}_{i}\left(\mathbf{q}_{i}\right)=\boldsymbol{\Psi}_{i}^{-1}\left(\mathbf{q}_{i}\right)$ are given by

$$
\boldsymbol{\Psi}_{i}\left(\mathbf{q}_{i}\right)=\left[\begin{array}{cc}
\sqrt{m_{11_{i}}-\frac{m_{12_{i}}^{2}}{m_{22_{i}}}} & 0 \\
\frac{m_{12_{i}}}{\sqrt{m_{22_{i}}}} & \sqrt{m_{22_{i}}}
\end{array}\right], \quad \mathbf{L}_{i}\left(\mathbf{q}_{i}\right)=\left[\begin{array}{cc}
\frac{1}{\Psi_{11_{i}}} & 0 \\
-\frac{\Psi_{21_{i}}}{\Psi_{11_{i} \Psi_{22_{i}}}} & \frac{1}{\Psi_{11_{i}}}
\end{array}\right],
$$

where $m_{j k_{i}}$ and $\Psi_{j k_{i}}$ are the $j k$-element of the matrices $\mathbf{M}_{i}\left(\mathbf{q}_{i}\right)$ and $\boldsymbol{\Psi}_{i}\left(\mathbf{q}_{i}\right)$, respectively. Note that the mapping $\boldsymbol{\Psi}_{i}\left(\mathbf{q}_{i}\right)$ satisfies $\mathbf{M}_{i}\left(\mathbf{q}_{i}\right)=\boldsymbol{\Psi}_{i}^{\top}\left(\mathbf{q}_{i}\right) \boldsymbol{\Psi}_{i}\left(\mathbf{q}_{i}\right)$, as required. The skew-symmetric matrix $\mathbf{S}_{i}\left(\mathbf{q}_{i}, \mathbf{x}_{i}\right)$ and matrix $\overline{\mathbf{S}}_{i}\left(\mathbf{q}_{i}, \boldsymbol{\xi}_{i}+\boldsymbol{\beta}_{i}\right)$ are

$$
\mathbf{S}_{i}\left(\mathbf{q}_{i}, \mathbf{x}_{i}\right)=\left[\begin{array}{cc}
0 & p_{1_{i}}\left(y_{2_{i}}\right) x_{1_{i}}+p_{2_{i}}\left(y_{2_{i}}\right) x_{2_{i}} \\
-p_{1_{i}}\left(y_{2_{i}}\right) x_{1_{i}}-p_{2_{i}}\left(y_{2_{i}}\right) x_{2_{i}} & 0
\end{array}\right]
$$


and

$$
\overline{\mathbf{S}}_{i}\left(\mathbf{q}_{i}, \boldsymbol{\xi}_{i}+\boldsymbol{\beta}_{i}\right)=\left[\begin{array}{cc}
p_{1_{i}}\left(q_{2_{i}}\right)\left(\xi_{2_{i}}+\beta_{2_{i}}\right) & p_{2_{i}}\left(q_{2_{i}}\right)\left(\xi_{2_{i}}+\beta_{2_{i}}\right) \\
-p_{1_{i}}\left(q_{2_{i}}\right)\left(\xi_{1_{i}}+\beta_{1_{i}}\right) & -p_{2_{i}}\left(q_{2_{i}}\right)\left(\xi_{1_{i}}+\beta_{1_{i}}\right)
\end{array}\right],
$$

where $\quad p_{1_{i}}\left(q_{2_{i}}\right)=\frac{\beta_{i} \mathrm{~s}_{2_{i}}}{\Psi_{11_{i}}^{2} \Psi_{22_{i}}}\left(1-\frac{\Psi_{21_{i}}}{\Psi_{22_{i}}}\right)$ and $p_{2_{i}}\left(q_{2_{i}}\right)=\frac{\beta_{i} \mathrm{~s}_{2_{i}}}{\Psi_{11_{i}} \Psi_{22_{i}}^{2}}$. Finally, the mappings $\mathbf{H}_{i}\left(\hat{\mathbf{q}}_{i}, \hat{\mathbf{x}}_{i}\right), \boldsymbol{\Delta}_{\mathbf{q}_{i}}, \boldsymbol{\Delta}_{\mathbf{x}_{i}}$ are given by

$$
\begin{gathered}
\mathbf{H}_{i}\left(\hat{\mathbf{q}}_{i}, \hat{\mathbf{x}}_{i}\right)=\left[\begin{array}{cc}
k \Psi_{11_{i}}\left(\hat{q}_{2_{i}}\right)+p_{3_{i}}\left(\hat{q}_{2_{i}}\right) \hat{x}_{2_{i}} & \Psi_{22_{i}} p_{2_{i}}\left(\hat{q}_{2_{i}}\right) \hat{x}_{2_{i}} \\
k \Psi_{21_{i}}\left(\hat{q}_{2_{i}}\right)-p_{3_{i}}\left(\hat{q}_{2_{i}}\right) \hat{x}_{1_{i}} & K \Psi_{22_{i}}-\Psi_{22_{i}} p_{2_{i}}\left(\hat{q}_{2_{i}}\right) \hat{x}_{1_{i}}
\end{array}\right], \\
\boldsymbol{\Delta}_{\mathbf{q}_{i}}=\left[\begin{array}{cc}
k\left[\Psi_{11_{i}}\left(q_{2_{i}}\right)-\Psi_{11_{i}}\left(\hat{q}_{2_{i}}\right)\right]+\left[p_{3_{i}}\left(q_{2_{i}}\right)-p_{3_{i}}\left(\hat{q}_{2_{i}}\right)\right] \hat{x}_{2_{i}} & \Psi_{22_{i}}\left[p_{2_{i}}\left(q_{2_{i}}\right)-p_{2_{i}}\left(\hat{q}_{2_{i}}\right)\right] \hat{x}_{2_{i}} \\
k\left[\Psi_{21_{i}}\left(q_{2_{i}}\right)-\Psi_{21_{i}}\left(\hat{q}_{2_{i}}\right)\right]-\left[p_{3_{i}}\left(q_{2_{i}}\right)-p_{3_{i}}\left(\hat{q}_{2_{i}}\right)\right] \hat{x}_{1_{i}} & \Psi_{22_{i}}\left[p_{2_{i}}\left(\hat{q}_{2_{i}}\right)-p_{2_{i}}\left(q_{2_{i}}\right)\right] \hat{x}_{1_{i}}
\end{array}\right]
\end{gathered}
$$

and

$$
\boldsymbol{\Delta}_{\mathbf{x}_{i}}=\left[\begin{array}{cc}
-p_{33_{i}}\left(q_{2_{i}}\right) e_{x_{2_{i}}} & -\Psi_{22_{i}} p_{2_{i}}\left(q_{2_{i}}\right) e_{x_{2_{i}}} \\
p_{3_{i}}\left(q_{2_{i}}\right) e_{x_{1_{i}}} & \Psi_{22_{i}} p_{2_{i}}\left(q_{2_{i}}\right) e_{x_{1_{i}}}
\end{array}\right],
$$

where $p_{3 i}\left(q_{2_{i}}\right)=\Psi_{11_{i}}\left(q_{2_{i}}\right) p_{1_{i}}\left(q_{2_{i}}\right)+\Psi_{21_{i}}\left(q_{2_{i}}\right) p_{2_{i}}\left(q_{2_{i}}\right)$. Clearly, $\mathbf{e}_{\mathbf{x}_{i}}=\mathbf{e}_{\mathbf{x}_{i}}=\mathbf{0}$ implies that $\boldsymbol{\Delta}_{\mathbf{q}_{i}}=$ $\boldsymbol{\Delta}_{\mathbf{x}_{i}}=\mathbf{0}$, as required.

The physical parameters for the manipulators are: $l_{1_{i}}=0.4 \mathrm{~m}, l_{2_{i}}=0.3 \mathrm{~m}, m_{1_{l}}=1.5 \mathrm{~kg}, m_{2_{l}}=$ $1 \mathrm{~kg}, m_{1_{r}}=2.5 \mathrm{~kg}$ and $m_{2_{r}}=1.5 \mathrm{~kg}$. The human operator is modeled as a spring-damper system $\boldsymbol{\tau}_{h}=K_{h}\left(\mathbf{q}_{h}-\mathbf{q}_{l}\right)-K_{d} \dot{\mathbf{q}}_{l}$ where $K_{h}=10 \mathrm{Nm}$ and $K_{d}=1 \mathrm{Nm} / \mathrm{s}$. Figure 2 depicts the desired human position $\mathbf{q}_{h}$. The initial conditions are $\dot{\mathbf{q}}_{i}(0)=\mathbf{0}, \mathbf{q}_{l}^{\top}(0)=[-0.5 \pi ; 0], \mathbf{q}_{r}^{\top}(0)=$ $[-0.25 \pi ; 0.15 \pi]$. All the observer state has zero initial conditions, except for $r_{i}(0)=1$.

The transmission of the local and remote positions, emulates a typical Internet User Datagram Protocol (UDP) setup. The transmission delays follow normal Gaussian distributions [31] and are depicted in Figure 3. The statistical parameters of these delays are: $0.2 \mathrm{~s}, 0.003 \mathrm{~s}$ and $0.3 \mathrm{~s}$ for the mean, variance and seed, respectively, for the local manipulator and $0.4 \mathrm{~s}, 0.004 \mathrm{~s}$ and $0.5 \mathrm{~s}$ for the mean, variance and seed, respectively, for the remote manipulator. The practical upper-bounds of these time-delays are: ${ }^{*} T_{l}=0.3 \mathrm{~s}$ and ${ }^{*} T_{r}=0.5 \mathrm{~s}$.

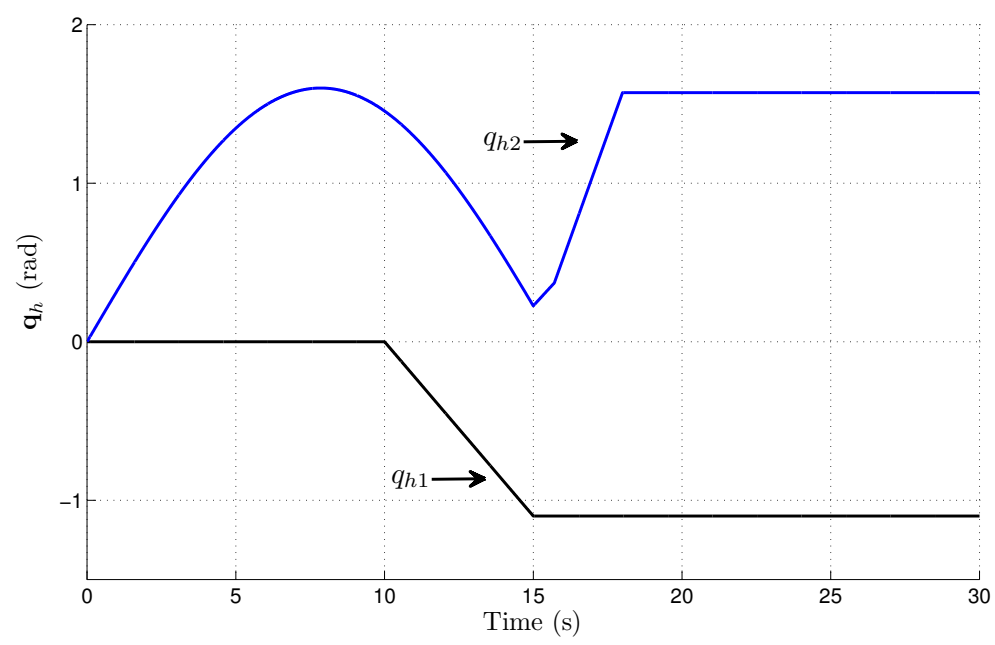

Figure 2. Desired human operator position. 


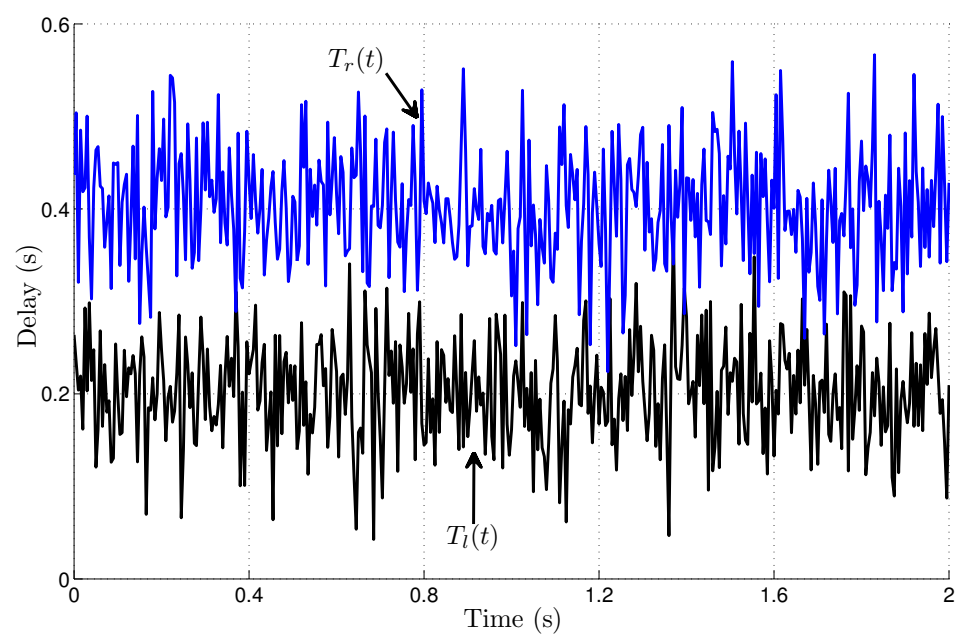

Figure 3. Variable time-delays in the local and remote interconnection.

For simplicity, the local and remote observer gains are equal and have been set as: $c_{1}=c_{2}=c_{3}=$ 5. The local and remote controller gains are set, in order to fulfill (30), as: $K_{l}=5, K_{r}=6, B_{l}=4$ and $B_{r}=5$.

Three different simulations have been performed: one in which the remote manipulator moves freely in its environment; another in which the remote manipulator interacts with a stiff wall and, finally, a position performance comparison between using the estimated velocities and the measured velocities in controller (22).

\subsection{Remote Manipulator in Free Space}

Figures 4 and 5 depict, in joint and Cartesian space, respectively, the local and remote position tracking capabilities when the remote manipulator moves freely. Figure 6 shows the behavior of the estimated velocities and the estimation error. From these figures it is concluded that, despite variable time-delays and without measuring joint velocities, position tracking is achieved and, as expected, the estimation of the velocities exponentially converge to the real velocity values.

\subsection{Remote Manipulator Interacting with a Wall}

In this set of simulations, a stiff wall is added in the remote environment. The wall is located in the $x y$-plane at $y=0.1 \mathrm{~m}$ and it is modeled as a spring-damper Cartesian system with stiffness equal to $20000 \mathrm{Nm}$ and damping equal to $10 \mathrm{Nm} / \mathrm{s}$.

Figures 7 and 8 show the local and remote joint and Cartesian positions, respectively. The remote manipulator becomes in contact with the wall from, around, $2 \mathrm{~s}$ to $12.5 \mathrm{~s}$. Despite such interaction and interconnecting delays, position error asymptotically converges to zero. Furthermore, as seen in Figure 9, the estimated velocities exponentially converge to the real ones. From Figure 10, that shows the human operator and the environment forces, it can be concluded that force tracking performance is not as superior as position tracking. 

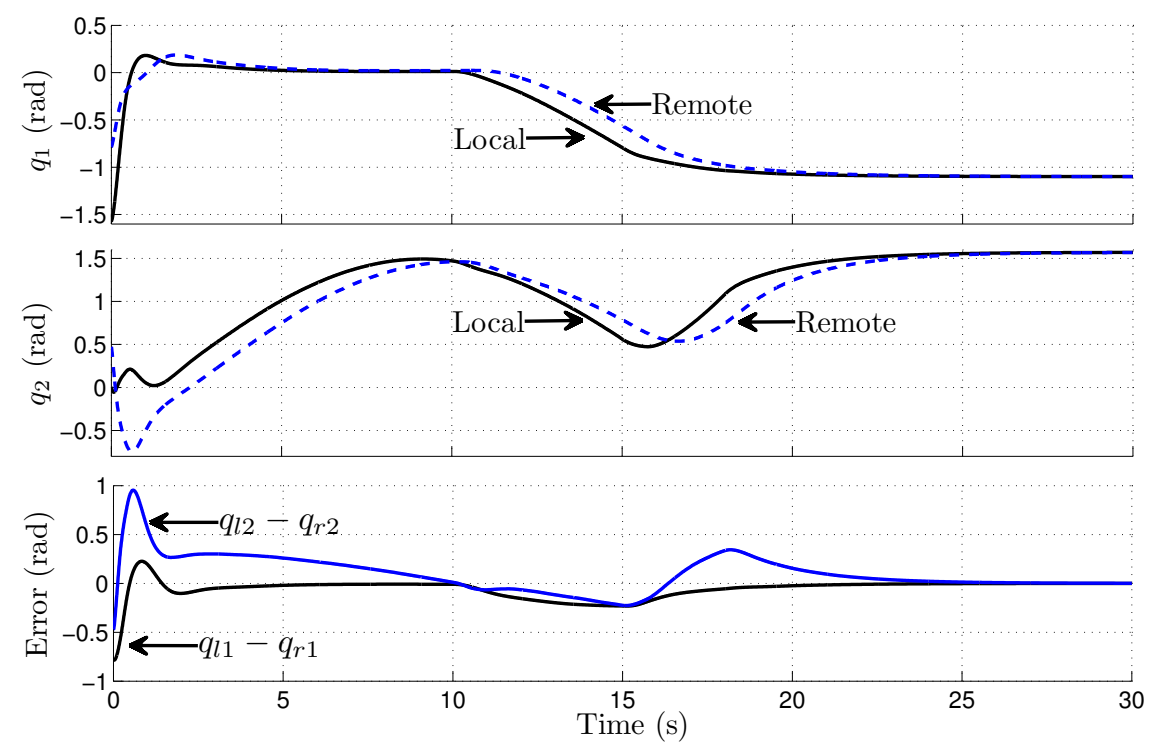

Figure 4. Local and remote positions in free space.
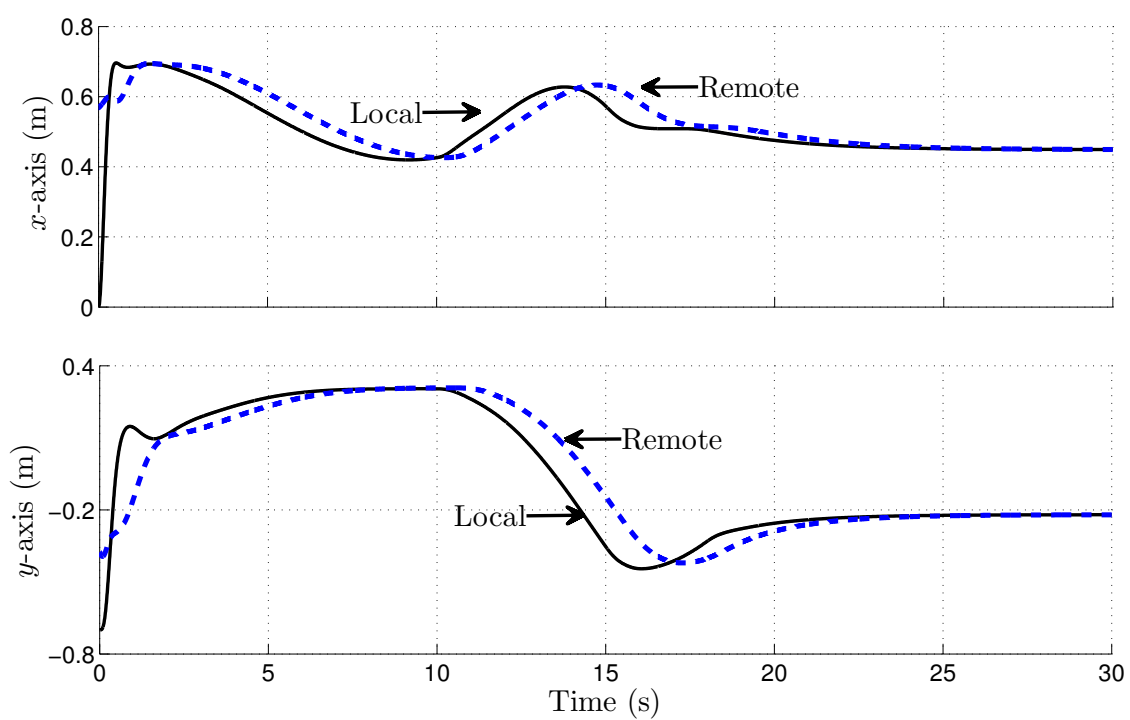

Figure 5. Local and remote Cartesian positions in free space.

\subsection{Performance Comparison}

This section compares the position performance of the simulated bilateral teleoperator, controlled by (22), using the estimated velocity feedback or the measured velocity feedback. In the latter, these controllers become

$$
\begin{aligned}
& \boldsymbol{\tau}_{l}=K_{l}\left[\mathbf{q}_{l}-\mathbf{q}_{r}\left(t-T_{r}(t)\right)\right]+B_{l} \mathbf{L}_{l}\left(\mathbf{q}_{l}\right) \mathbf{x}_{l}-\mathbf{g}_{l} \\
& \boldsymbol{\tau}_{r}=-K_{r}\left[\mathbf{q}_{r}-\mathbf{q}_{l}\left(t-T_{l}(t)\right)\right]-B_{r} \mathbf{L}_{r}\left(\mathbf{q}_{r}\right) \mathbf{x}_{r}+\mathbf{g}_{r} .
\end{aligned}
$$

All the system parameters and the controller gains are the same for both controllers. In this scenario, both remote interaction cases, free space and stiff-wall interaction, have been simulated. 


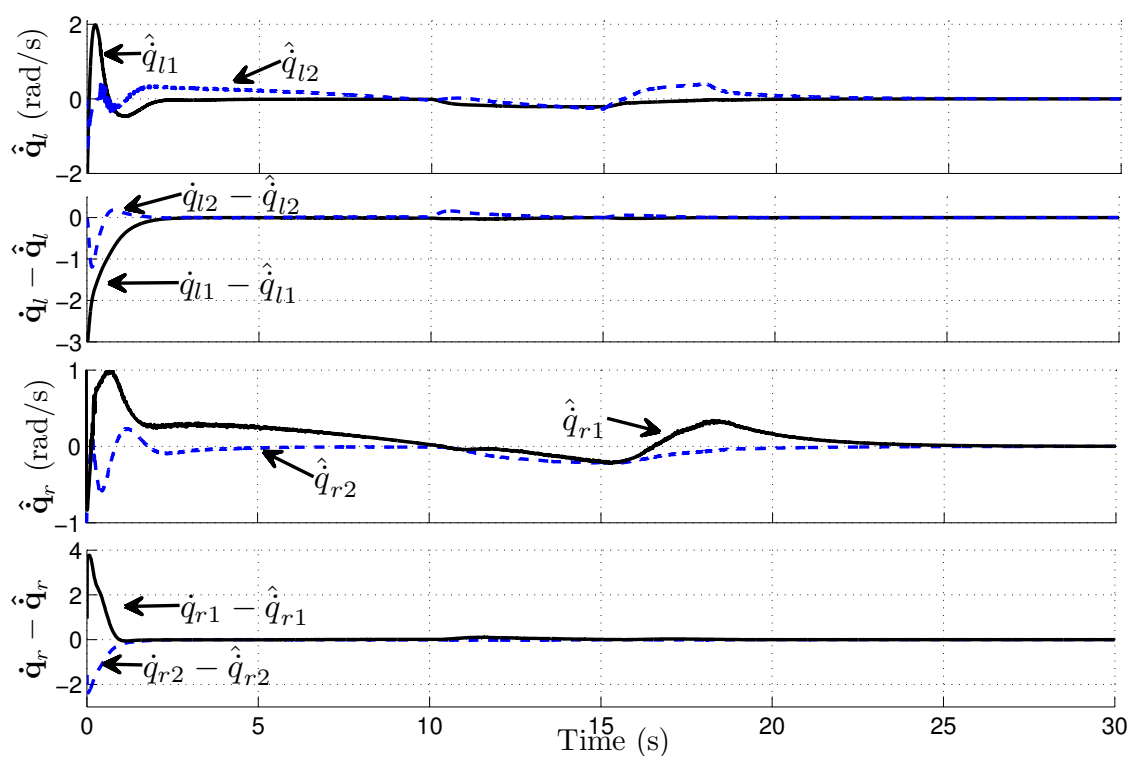

Figure 6. Local and remote estimated velocities in free space.

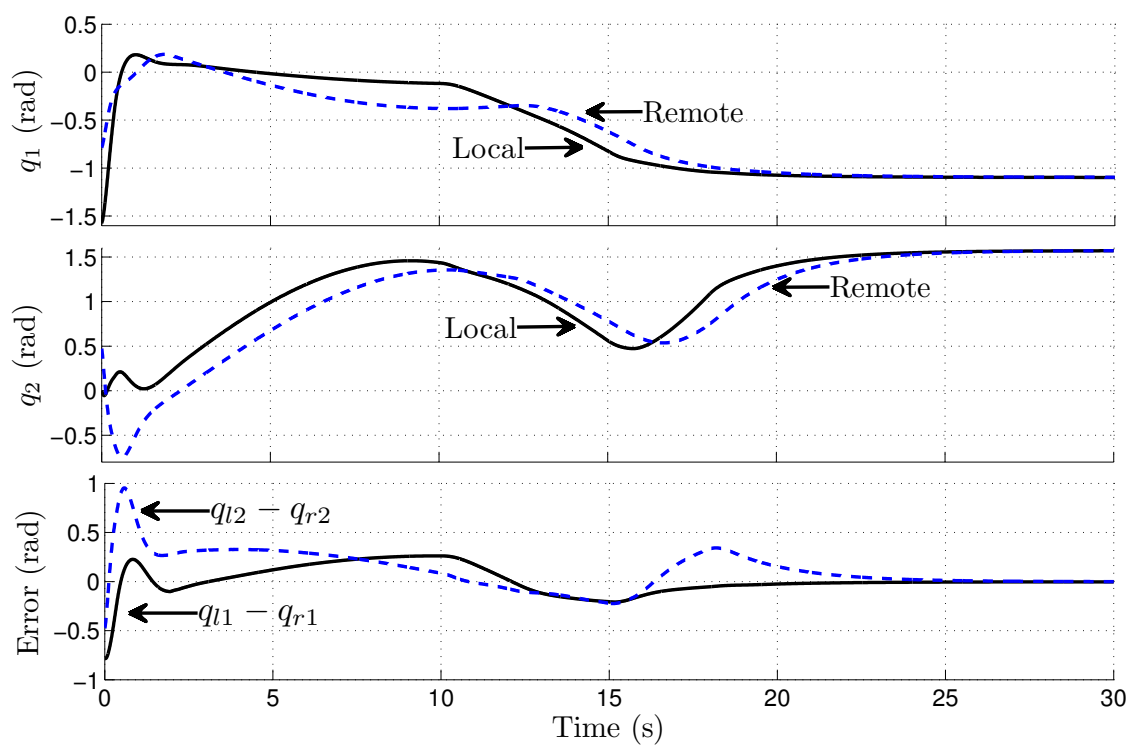

Figure 7. Local and remote articular positions when interacting with a remote wall.

Figures 11 and 12 show the position performance comparison when the remote manipulator moves freely in the space. After the transient stage, the position response of both schemes is, practically, the same. From Figure 6, it can be seen that such transient lasts for two seconds. The same behavior conclusions can be drawn for the case when the remote manipulator interacts with a stiff-wall, in Figures 13 and 14. The stiffness and the wall position are kept the same as before. 

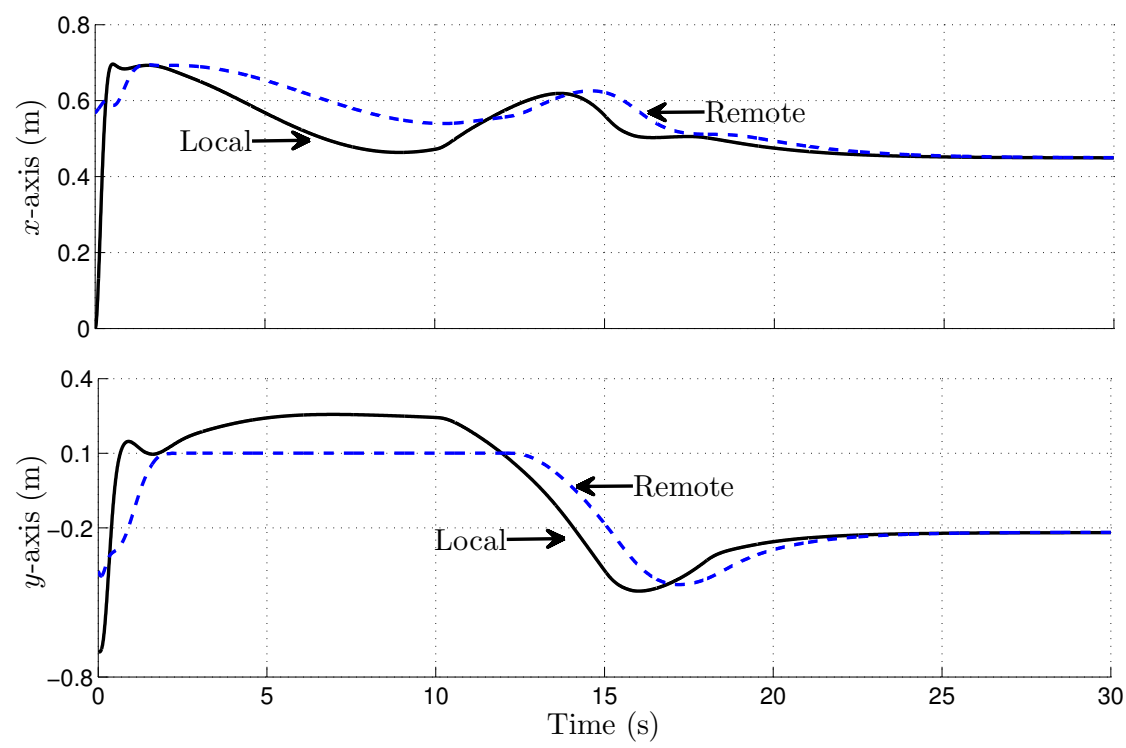

Figure 8. Local and remote Cartesian position when interacting with a remote wall.
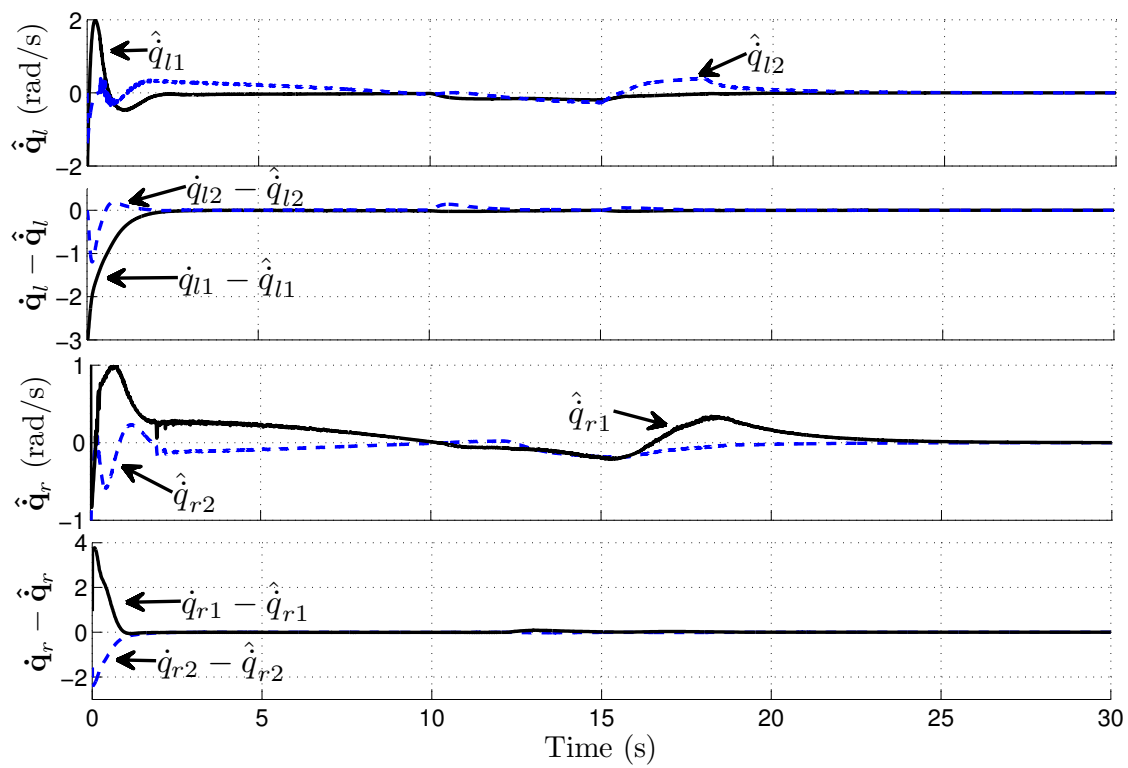

Figure 9. Local and remote estimated velocities when interacting with a remote wall.

\section{CONCLUSIONS AND FUTURE WORK}

A solution to the position tracking problem for bilateral teleoperators with variable time-delays and with unmeasured velocities is reported. Such solution exploits the immersion and invariance observer, in order to obtain a globally exponentially convergent estimate of the unmeasured velocities. Through a Lyapunov-like analysis, it is proved that this observer in conjunction with a simple P+d controller ensures the global boundedness of the closed-loop trajectories, provided that the human and the environment are passive. Furthermore, in the absence of human and remote forces, position coordination with zero velocities is established. Simulations and a performance 

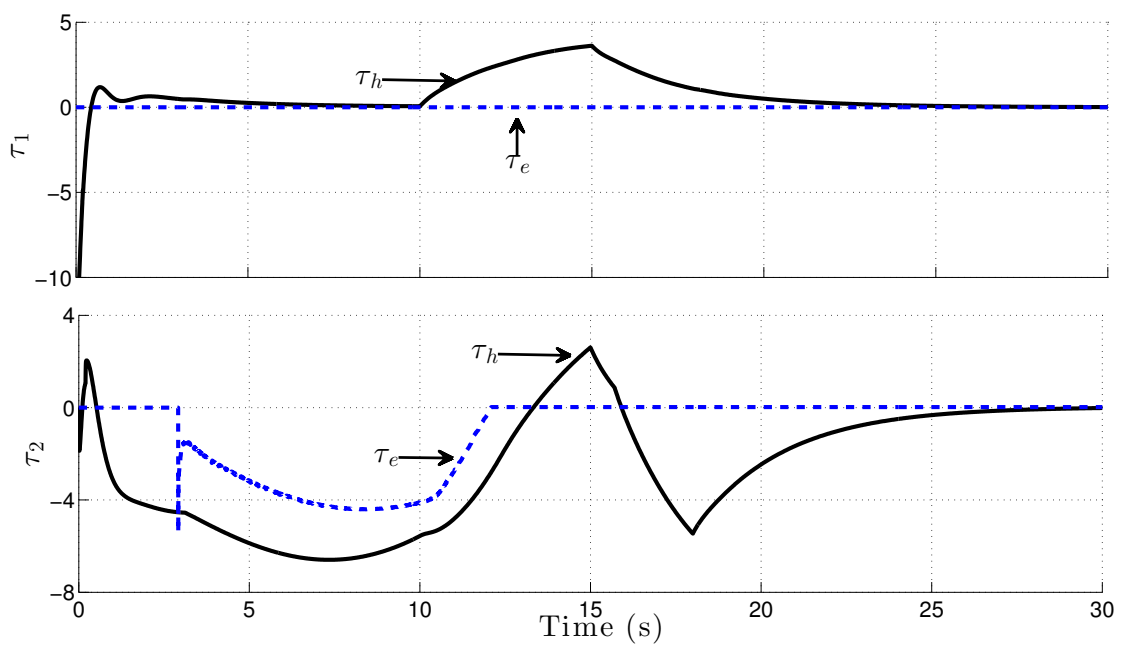

Figure 10. Human operator and environment forces.
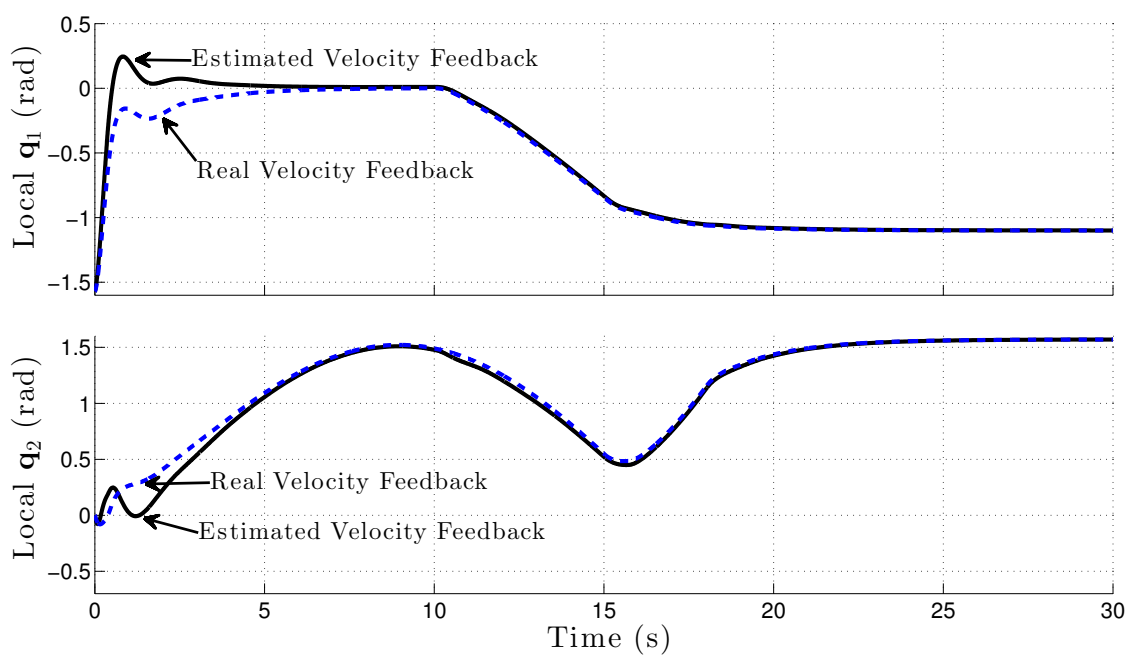

Figure 11. Local position performance comparison in free space.
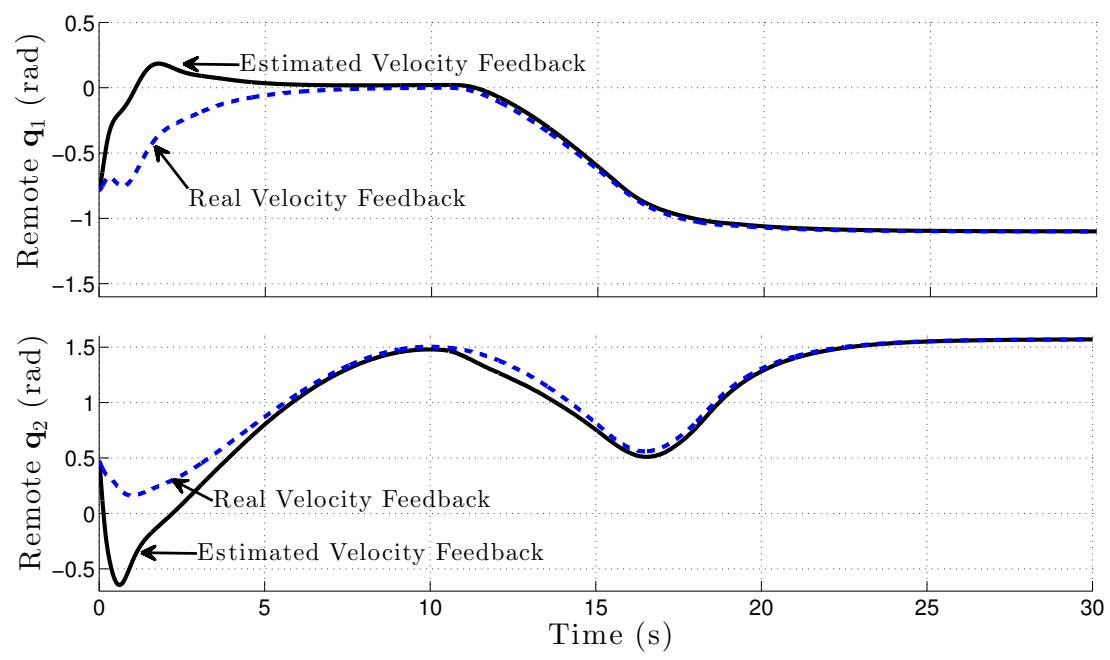

Figure 12. Remote position performance comparison in free space. 

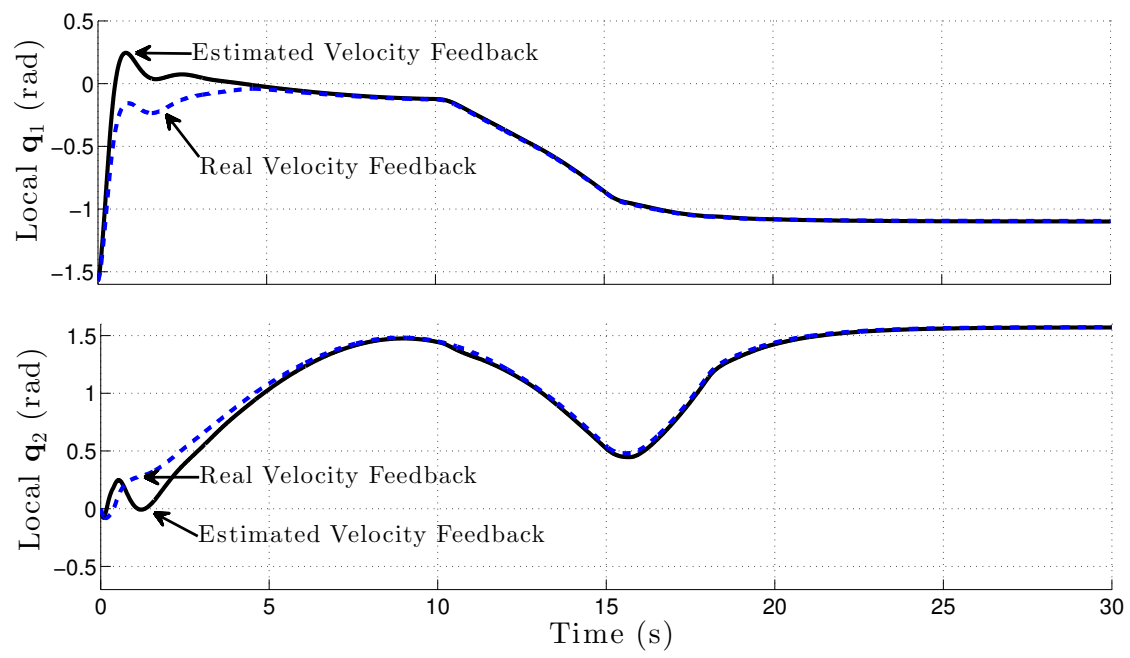

Figure 13. Local position performance comparison with the remote stiff-wall interaction.
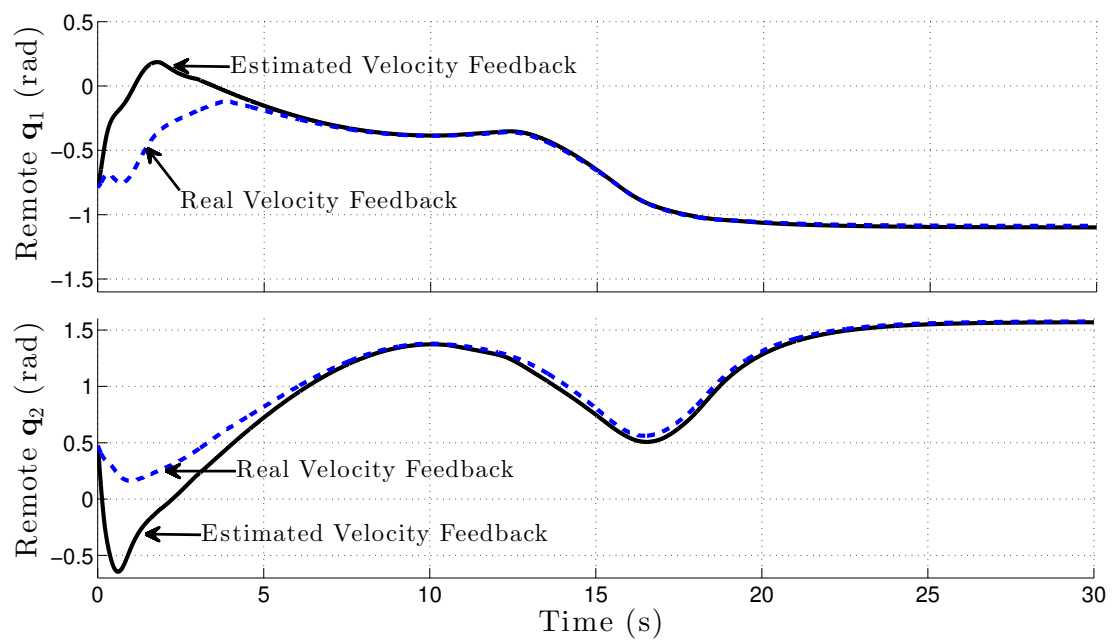

Figure 14. Remote position performance comparison with the remote stiff-wall interaction.

comparison, using a couple of 2-DOF nonlinear manipulator are presented to illustrate the behavior of the complete observer plus controller system.

Future research will focus on extending this proposal to bilateral teleoperators with flexible joint manipulators. Another research avenue is the extension of this work to the case of the consensus control in networks of Euler-Lagrange systems with interconnecting delays.

\section{ACKNOWLEDGEMENTS}

This work has been partially supported by the Mexican projects CONACyT CB-129079 and INFR-229696, the Spanish PN I+D+i projects DPI2013-40882-P and DPI2011-22471 and the IAP Programme initiated by the Belgian State, Science Policy Office, through the Belgian Network DYSCO. I. Sarras expresses his gratitude to the members of the Institute of Industrial and 
Control Engineering at the Technical University of Catalonia for their hospitality. The scientific responsibility rests with the authors.

\section{REFERENCES}

1. Anderson R, Spong M. Bilateral control of teleoperators with time delay. IEEE Transactions on Automatic Control May 1989; 34(5):494-501.

2. Niemeyer G, Slotine J. Stable adaptive teleoperation. IEEE Journal of Oceanic Engineering Jan 1991; 16(1):152162.

3. Hokayem P, Spong M. Bilateral teleoperation: An historical survey. Automatica 2006; 42:2035-2057.

4. Nuño E, Basañez L, Ortega R. Passivity-based control for bilateral teleoperation: A tutorial. Automatica 2011; 47(3):485-495.

5. Chopra N, Spong M, Ortega R, Barbanov N. On tracking preformance in bilateral teleoperation. IEEE Transactions on Robotics Aug 2006; 22(4):844-847.

6. Lee D, Spong M. Passive bilateral teleoperation with constant time delay. IEEE Transactions on Robotics April 2006; 22(2):269-281.

7. Nuño E, Ortega R, Barabanov N, Basañez L. A globally stable PD controller for bilateral teleoperators. IEEE Transactions on Robotics 2008; 24(3):753-758.

8. Nuño E, Ortega R, Basañez L. An adaptive controller for nonlinear bilateral teleoperators. Automatica 2010; 46(1):155-159.

9. Nuño E, Ortega R, Basañez L, Hill D. Synchronization of networks of nonidentical Euler-Lagrange systems with uncertain parameters and communication delays. IEEE Transactions on Automatic Control 2011; 56(4):935-941.

10. Aldana C, Nuño E, Basañez L. Bilateral teleoperation of cooperative manipulators. IEEE International Conference on Robotics and Automation (ICRA), Saint Paul, MN, USA, 2012; 4274-4279.

11. Nuño E, Basañez L, Ortega R, Spong M. Position tracking for nonlinear teleoperators with variable time-delay. The International Journal of Robotics Research 2009; 28(7):895-910.

12. Nuño E, Sarras I, Basañez L. Consensus in networks of nonidentical Euler-Lagrange systems using P+d controllers. IEEE Transactions on Robotics 2013; 26(6):1503-1508.

13. Nuño E, Ortega R, Jayawardhana B, Basañez L. Coordination of multi-agent Euler-Lagrange systems via energyshaping: Networking improves robustness. Automatica 2013; 49(10):3065-3071.

14. Stramigioli S, van der Schaft A, Maschke B, Melchiorri C. Geometric scattering in robotic telemanipulation. IEEE Transactions on Robotics and Automation Aug 2002; 18(4):588-596.

15. Arcara P, Melchiorri C. Control schemes for teleoperation with time delay: A comparative study. Robotics and Autonomous Systems Jan 2002; 38(1):49-64.

16. Secchi C, Stramigioli S, Fantuzzi C. Transparency in port-hamiltonian-based telemanipulation. IEEE Transactions on Robotics Aug 2008; 24(4):903-910.

17. Chopra N, Spong M, Lozano R. Synchronization of bilateral teleoperators with time delay. Automatica August 2008; 44(8):2142-2148.

18. Garcia-Valdovinos L, Parra-Vega V, Arteaga M. Observer-based sliding mode impedance control of bilateral teleoperation under constant unknown time delay. Robotics and Autonomous Systems Aug 2007; 55(8):609-617.

19. Polushin I, Tayebi A, Marquez H. Control schemes for stable teleoperation with communication delay based on ios small gain theorem. Automatica June 2006; 42(6):905-915.

20. Hua $\mathrm{C}$, Liu X. Teleoperation over the internet with/without velocity signal. IEEE Transactions on Instrumentation and Measurement January 2011; 60(1):4-13.

21. Nuño E, Basañez L, López-Franco C, Arana-Daniel N. Stability of nonlinear teleoperators using PD controllers without velocity measurements. Journal of the Franklin Institute 2014; 351(1):241-258.

22. Astolfi A, Ortega R, Venkatraman A. A globally exponentially convergent immersion and invariance speed observer for mechanical systems with non-holonomic constraints. Automatica Jan 2010; 46(1):182-189.

23. Astolfi A, Ortega R, Venkatraman A. A globally exponentially convergent immersion and invariance speed observer for $\mathrm{n}$ degrees of freedom mechanical systems. 48th IEEE Conference on Decision and Control Dec 2009; :65086513.

24. Karagiannis D, Astolfi A. Observer design for a class of nonlinear systems using dynamic scaling with application to adaptive control. 47th IEEE Conference on Decision and Control Dec 2008; :2314-2319.

25. Astolfi A, Karagiannis D, Ortega R. Nonlinear and adaptive control design and applications. Springer-Verlag: London, 2007. 
26. Venkatraman A, Ortega R, Sarras I, der Schaft AV. Speed observation and position feedback stabilization of partially linearizable mechanical systems. IEEE Transactions on Automatic Control May 2010; 5(5):1059-1074.

27. Sarras I, Nuño E, Kinnaert M, Basañez L. Output-feedback control of nonlinear bilateral teleoperators. American Control Conference (ACC), IEEE (ed.), Montreal, CA, 2012; 3490-3495.

28. Kelly R, Santibáñez V, Loria A. Control of robot manipulators in joint space. Advanced textbooks in control and signal processing, Springer-Verlag, 2005.

29. Spong M, Hutchinson S, Vidyasagar M. Robot Modeling and Control. Wiley, 2005.

30. Lichiardopol S, van-de-Wouw N, Kostic D, Nijmeijer H. Trajectory tracking control for a tele-operation setup with disturbance estimation and compensation. 49th IEEE Conference on Decision and Control, Atlanta, USA, 2010; 1142-1147.

31. Salvo-Rossi P, Romano G, Palmieri F, Iannello G. Joint end-to-end loss-delay hidden markov model for periodic UDP traffic over the internet. IEEE Transactions on Signal Processing 2006; 54(2):530-541. 